\title{
A Three-Dimensional Trajectory Model with Advection Correction for Tropical Cyclones: Algorithm Description and Tests for Accuracy
}

\author{
WiLliam MiLler AND DA-LiN ZHANG \\ Department of Atmospheric and Oceanic Science, University of Maryland, College Park, College Park, Maryland
}

(Manuscript received 14 December 2018, in final form 25 April 2019)

\begin{abstract}
When computing trajectories from model output, gridded winds are often temporally interpolated to a time step shorter than model output intervals to satisfy computational stability constraints. This study investigates whether trajectory accuracy may be improved for tropical cyclone (TC) applications by interpolating the model winds using advection correction (AC) instead of the traditional linear interpolation in time (LI) method. Originally developed for Doppler radar processing, AC algorithms interpolate data in a reference frame that moves with the pattern translation, or advective flow velocity. A previously developed trajectory AC implementation is modified here by extending it to three-dimensional (3D) flows, and the advective flows are defined in cylindrical rather than Cartesian coordinates. This AC algorithm is tested on two model-simulated TC cases, Hurricanes Joaquin (2015) and Wilma (2005). Several variations of the AC algorithm are compared to LI on a sample of 10201 backward trajectories computed from the modeled 5-min output data, using reference trajectories computed from 1-min output to quantify position errors. Results show that $\mathrm{AC}$ of $3 \mathrm{D}$ wind vectors using advective flows defined as local gridpoint averages improves the accuracy of most trajectories, with more substantial improvements being found in the inner eyewall where the horizontal flows are dominated by rotating cyclonic wind perturbations. Furthermore, AC eliminates oscillations in vertical velocity along LI backward trajectories run through deep convective updrafts, leading to a $\sim 2.5-\mathrm{km}$ correction in parcel height after $20 \mathrm{~min}$ of integration.
\end{abstract}

\section{Introduction}

A Lagrangian, or parcel-following, analysis is a powerful tool for studying a wide variety of atmospheric processes. Applications include the tracking of ash clouds following volcanic eruptions (Kristiansen et al. 2012), studying ozone and water vapor exchange between the troposphere and stratosphere (Homeyer et al. 2011), pollutant transport and dispersion analysis (Stohl and Kromp-Kolb 1994; Stohl 1996; Brankov et al. 1998; Baker 2010), and investigating sources of updraft buoyancy in tropical maritime convection (Fierro et al. 2009, 2012). Some atmospheric models have a built-in capability to compute forward parcel trajectories during run time with the same computational time step used for integrating the prognostic governing equations (Fierro et al. 2009, 2012; Dahl et al. 2012). Nevertheless, for many problems, or if backward trajectories are needed, the four-dimensional (4D) gridded winds must be supplied by analyses or offline model runs. A fluid parcel

Corresponding author: Da-Lin Zhang, dalin@umd.edu may be uniquely identified by its position $\mathbf{x}_{0}$ at any initial, or "seed" time, $t_{0}$. The parcel position $\mathbf{x}\left(\mathbf{x}_{0}, t\right)$ at any time $t_{f}$ may be found by integrating

$$
D \mathbf{x} / D t=\mathbf{u}(\mathbf{x}, t)
$$

using the Lagrangian derivative with respect to time $(D / D t)$ over the time interval $\left[t_{0}, t_{f}\right]$, where $\mathbf{u}(\mathbf{x}, t)$ is the parcel velocity. Since parcels are free to travel between the grid vertices, $\mathbf{u}(\mathbf{x}, t)$ is obtained by interpolating the Eulerian gridded winds to the parcel position at every computational time step. A subclass of trajectory models, commonly referred to as Lagrangian dispersion models, accounts for parameterized subgrid scales of motion when solving for $\mathbf{u}(\mathbf{x}, t)$; however, this study focuses on trajectory applications where the unresolved motion scales may be neglected.

Model output temporal resolution is often limited by disk storage constraints; for these cases, Eq. (1) is typically integrated using a considerably smaller time step to minimize numerical truncation errors. Temporal interpolation of the gridded wind field to the computational times has been shown to be a significant trajectory error 
source (Kuo et al. 1985; Rössler et al. 1992; Stohl et al. 1995; Dahl et al. 2012). ${ }^{1}$ For example, Dahl et al. (2012) simulated a supercell thunderstorm using the Bryan Cloud Model 1 (CM1) and generated forward "truth" trajectories during model run time that passed through the near-surface mesocyclone. They ran backward trajectories from the forward trajectory end-points using varying model output time intervals, and found that applying linear time interpolation (LI) to the confluent, nonlinearly evolving flow resulted in fictitious low-level inflow trajectories for the temporally coarser input data. Trajectory models have traditionally used LI to estimate $\mathbf{u}(\mathbf{x}, t)$ from gridded input (Bowman et al. 2013). This method should be well suited for any velocity field with an approximately linear evolution between sampling times.

Shapiro et al. (2015, hereafter S15) tested an alternative time interpolation technique using advection correction (AC) on two-dimensional (2D) trajectories run from a high-resolution $(30 \mathrm{~m}) \mathrm{CM} 1$ supercell simulation. AC algorithms have been extensively used in Doppler radar data processing when an analysis reflectivity or wind field must be constructed from nonsimultaneous beam scan observations; they essentially redefine the observations in a reference frame moving with the pattern translation velocity (Gal-Chen 1982, hereafter GC82; Zhang and Gal-Chen 1996; Dowell and Bluestein 2002; Shapiro et al. 2010). Let us now illustrate, in a simple example, how AC can be applied to the time interpolation of the vertical velocity field; this concept can be extended to any scalar or Cartesian velocity vector component translating through three-dimensional (3D) space at constant velocity. Consider a wavelike updraft pattern $w(x, t)$ moving in the positive- $x$ direction with a speed $U\left(\mathrm{~m} \mathrm{~s}^{-1}\right)$, as shown in Fig. $1 ; w$ is perfectly resolved in space but not in time. Suppose that $w$ can be sampled at data input times $t=t_{0}$ and $t=t_{0}+\Delta t$, and we wish to find $w\left(p, t_{0}+\Delta t / 2\right)$ for $x=p$. Although the updraft peak passes $p$ at time $t=$ $t_{0}+\Delta t / 2$, it is displaced away from $p$ at both data input times, and hence LI between $w\left(p, t_{0}\right)$ and $w\left(p, t_{0}+\Delta t\right)$ yields an erroneously low estimate. Let us now redefine $p$ in a reference frame moving with velocity $U$ that is collocated with the fixed frame only for $t=t_{0}+\Delta t / 2$. For times $t=t_{0}$ and $t=t_{0}+\Delta t$, the positions of $p$ in the fixed reference frame, $p_{t 0}$ and $p_{t 0+\Delta t}$, respectively, are

$$
p_{t_{0}}=p-U \Delta t / 2
$$

\footnotetext{
${ }^{1}$ Other sources of trajectory error include the spatial interpolation of the gridded winds, numerical truncation errors accumulated from integrating Eq. (1), and inaccuracies in the gridded input winds themselves.
}

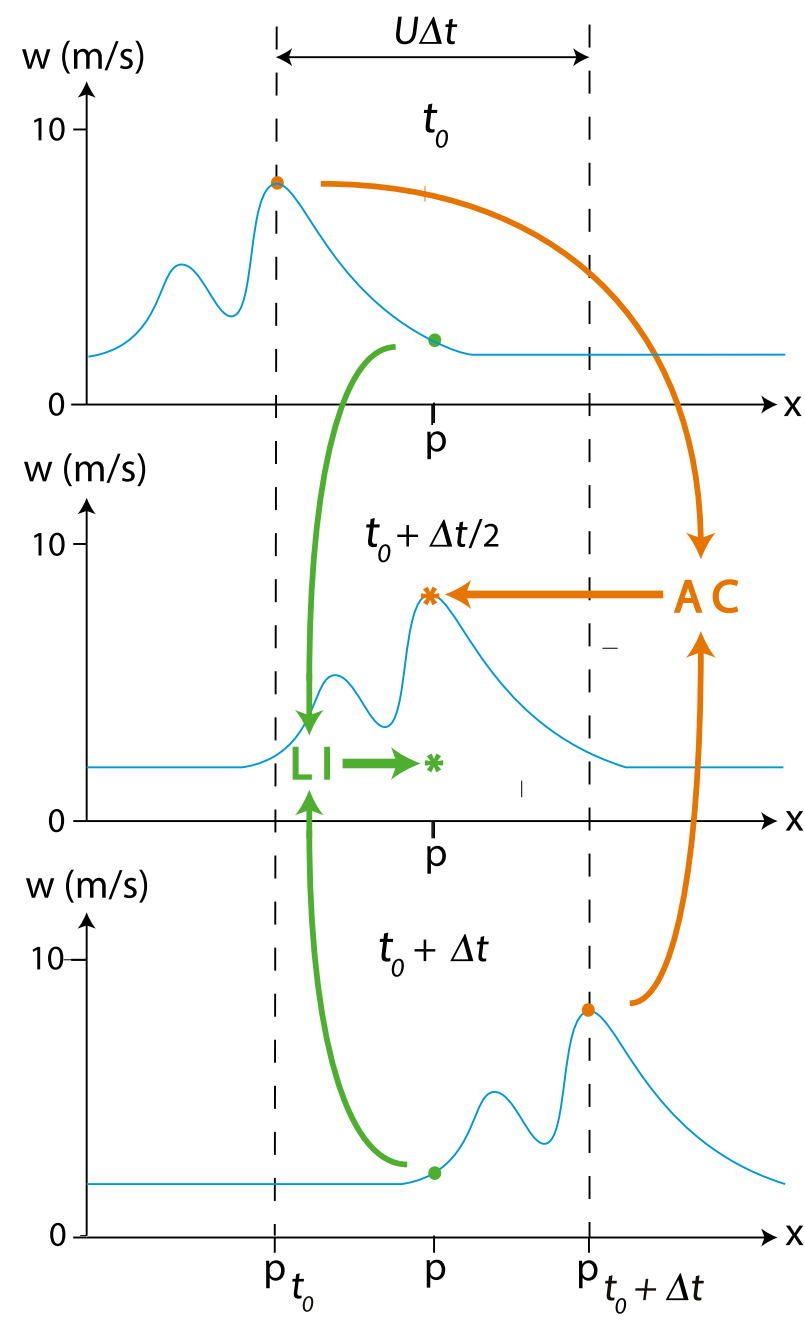

FIG. 1. Illustration of the advection correction principle for an updraft waveform $w(x, t)$ translating in the positive- $x$ direction with velocity $U$. At $x=p$, the waveform amplitude at time $t=t_{0}+\Delta t / 2$ is estimated by temporal interpolation between data input times $t=t_{0}$ and $t=t_{0}+\Delta t$. Simple linear interpolation (LI) in time from $x=p$ substantially underestimates $w$, whereas advection correction (AC) time interpolation from the updraft positions at times $t=t_{0}$ and $t=t_{0}+\Delta t$ more closely approximates the true $w$ amplitude as long as the waveform structure does not substantially change over time interval $\Delta t$.

$$
p_{t_{0}+\Delta t}=p+U \Delta t / 2
$$

For this simple case, $D w / D t=0$, and therefore $w\left(p, t_{0}+\right.$ $\Delta t / 2)$ is exactly equivalent to both $w\left(p_{t 0}, t_{0}\right)$ and $w\left(p_{t 0+\Delta t}\right.$, $\left.t_{0}+\Delta t\right)$. On the other hand, if the waveform were to grow or decay, LI between $w\left(p_{t 0}, t_{0}\right)$ and $w\left(p_{t 0+\Delta t}, t_{0}+\Delta t\right)$ could still improve the estimate of $w\left(p, t_{0}+\Delta t / 2\right)$ compared to LI from $x=p$, provided that the waveform retains its general shape. Herein LI will refer to linear time interpolation in the traditional sense (i.e., with no advection correction of the fixed frame coordinate position). 
S15 found that advection-correcting the 2D wind field improved the accuracy of most trajectories in their samples, relative to LI. However, they cautioned that defining the pattern translation velocities for realistic atmospheric flows could be a nontrivial task. According to the "frozen turbulence hypothesis" (Taylor 1938), a two-dimensional fluid vector field $(u, v)$ may be idealized in terms of a complex turbulent pattern $\left(u^{\prime}, v^{\prime}\right)$ that retains its shape over sufficiently short time intervals while translating with an "advective" flow vector $(U, V)$. Recognizing that many atmospheric features have advective flow patterns with spatiotemporal variation, S15 divided their model output domain into a checkerboard pattern of nonoverlapping "subdomains" and defined $U$ and $V$ for each subdomain and model output time interval. They tested two methods for determining the advective flows: (i) averaging the model-output winds over the subdomain, and (ii) using an "iterative GalChen procedure" developed by GC82 that yielded an advection-corrected $u$ field with the smallest departure from Lagrangian conservation following $U$ and $V$. The latter method minimized a cost function that penalized deviations from the frozen turbulence constraint, yielding linear equations for $U$ and $V$ in terms of local $u$ gradients and time tendencies integrated over the subdomain. AC using $U$ and $V$ determined by this method yielded the most accurate trajectories. S15 also showed how the iterative Gal-Chen procedure could converge on spurious $U$ and $V$ solutions if the input data temporal resolution became too coarse.

The major objective of this study is to assess the utility of $\mathrm{AC}$ in reducing time interpolation errors for trajectories computed from tropical cyclone (TC) simulation output. To accomplish this, we extend the 2D AC algorithm developed by S15 to 3D. Unlike S15, we define the advection velocities in cylindrical coordinates $(r, \lambda, z)$, where $r, \lambda$, and $z$ are radius, azimuthal angle, and height, respectively; this is a convenient framework for analyzing the swirling wind circulations of TCs. Radial velocity $u_{r}=$ $d r / d t$ and angular velocity $\omega=d \lambda / d t$ can be expressed as

$$
\begin{gathered}
u_{r}=U_{r}+u_{r}^{\prime}, \\
\omega=\Omega+\omega^{\prime},
\end{gathered}
$$

where capital letters and primes denote advective and perturbation components, respectively. Here, advective flows are defined over nonoverlapping subdomains on the computational grid for every model output time interval. We evaluate how AC impacts trajectory accuracy with $U_{r}$ and $\Omega$ determined by either (i) a gridpoint spatiotemporal average over each subdomain or (ii) a modified version of the S15 iterative Gal-Chen procedure that solves for the advective flows $U_{r}$ and $\Omega$ based on the absolute angular momentum (AAM) conservation principle. For axisymmetric TCs, it is well established that AAM, defined in terms of radius $r$ measured from the vortex center, tangential wind $v_{t}=\omega r$, and Coriolis parameter $f$ :

$$
\mathrm{AAM}=r\left(v_{t}+f r / 2\right),
$$

is nearly conserved following the flow above the maritime boundary layer (MBL), where friction and diffusion may be neglected (Zhang et al. 2001; Montgomery and Smith 2014; Qin et al. 2018). Here we invoke the additional assumption that the pressure torque $\partial p / \partial \lambda$ (Zhang et al. 2001) is small enough such that AAM can be treated as a "quasi-conserved" variable following 3D flows over sufficiently short time intervals:

$$
\begin{aligned}
\frac{D \mathrm{AAM}}{D t}= & \frac{\partial \mathrm{AAM}}{\partial t}+U_{r} \frac{\partial \mathrm{AAM}}{\partial r} \\
& +\Omega \frac{\partial \mathrm{AAM}}{\partial \lambda}+W \frac{\partial \mathrm{AAM}}{\partial z} \approx 0 .
\end{aligned}
$$

We will test this algorithm on trajectories run from the Advanced Research version of the Weather Research and Forecasting (WRF-ARW) Model simulations of Hurricanes Joaquin (2015) and Wilma (2005).

TC simulations offer a promising test bed for trajectory AC algorithms. Advances in observing systems and high-resolution modeling over the past 20 years have revealed that TC eyewall swirling winds have a rich and complex kinematic structure, with various types of embedded flow disturbances, even for mature, relatively axisymmetric cases. Outbreaks of deep convection, known as hot towers or convective bursts (CBs), have been shown to precede or accompany TC intensification episodes (Heymsfield et al. 2001; Guimond et al. 2010; Chen and Zhang 2013 hereafter CZ13; Rogers et al. 2015). A few previous studies have examined TC dynamical processes in a Lagrangian framework. Perhaps Liu et al. (1999) was the first to show the long residence of air parcels in the eye of their MM5-simulated Hurricane Andrew (1992) through forward and backward trajectories that were calculated by interpolating hourly model outputs into 3-min intervals. Using trajectories run from his MM5-simulated Hurricane Bob (1991) outputs at 2-min intervals, Braun (2002) showed that perturbation pressure gradient forces lifted air parcels above the eyewall MBL, with the parcels acquiring thermal buoyancy at higher levels. Cram et al. (2007) studied mixing between the eyewall, eye, and outer regions using trajectories computed from a Hurricane Bonnie (1998) MM5 simulation output at 3-min resolution. Their analysis revealed that while high-equivalent 
potential temperature $\left(\theta_{e}\right)$ air parcels from the low-level eye can be mixed into eyewall updrafts, providing a source of enhanced buoyancy, this sheared TC also experienced a $\sim 1-\mathrm{K}$ mean eyewall $\theta_{e}$ reduction due to extensive mixing of low $-\theta_{e}$ parcels from outer regions. Stern and Zhang (2013) investigated eye warming in an idealized WRF simulation using backward trajectories generated by the RIP4 postprocessing software (Stoelinga 2009) from 1- and 6-min output data. They found that once a threshold intensity of $\sim 40 \mathrm{~m} \mathrm{~s}^{-1}$ is reached, stirring between the eye and eyewall becomes reduced to the extent that upper-level parcels originating near the eye center can remain there for several days and descend $5-10 \mathrm{~km}$, even in the presence of moderate vertical wind shear (VWS). Onderlinde and Nolan (2016) used trajectories computed from 1-min resolution WRF idealized simulation output to show how the faster (slower) intensification rate of a vertically sheared TC with positive (negative) TC-relative environmental helicity resulted in part from a larger (smaller) percentage of boundary layer parcels being lofted into deep convective updrafts in upshear quadrants.

The next section describes the WRF simulation datasets and trajectory computation, AC, and trajectory error diagnostics methods. Section 3 shows a series of analytical trajectory tests to make sure that our codes are free of errors. Sections 4 and 5 compare the impacts of $\mathrm{AC}$ and LI on trajectories using the Hurricanes Joaquin (2015) and Wilma (2005) simulations, respectively. A summary and the conclusions are given in the final section. Appendixes A and B describe the vortex center-finding technique and computational considerations, respectively.

\section{Datasets and methodology}

\section{a. WRF simulation datasets}

Hurricane Wilma (2005) underwent a record-breaking 18-h rapid intensification (RI) episode on 18-19 October that culminated in a peak intensity in terms of minimum central sea level pressure $\left(P_{\mathrm{MIN}}\right)$ and maximum surface wind $\left(V_{\mathrm{MAX}}\right)$ of $882 \mathrm{hPa}$ and $82 \mathrm{~ms}^{-1}$, respectively (Pasch et al. 2006; Chen et al. 2011). RI is defined herein by the $V_{\text {MAX }}$ intensification exceeding $15 \mathrm{~m} \mathrm{~s}^{-1}(24 \mathrm{~h})^{-1}$ (Kaplan and DeMaria 2003). At peak intensity, Wilma (2005) was also the strongest hurricane ever recorded in the Atlantic basin. Wilma intensified in the western Caribbean under near-ideal environmental conditions with low VWS and high sea surface temperatures (SSTs) of $29^{\circ}-30^{\circ} \mathrm{C}$. Chen et al. (2011) simulated this storm using the WRF-ARW Model with a quadruply nested 27/9/3/1-km two-way interactive grid, 55 vertical $\sigma$ levels, and a 30-hPa model top. The 1-km nest, with $(x, y)$ grid dimensions of $451 \times 451$, followed the vortex center. This simulation captured the timing and rate of Wilma's RI reasonably well, and two subsequent papers (CZ13; Miller et al. 2015) showed how extreme upper-level CB updrafts exceeding $15 \mathrm{~m} \mathrm{~s}^{-1}$ may have contributed to Wilma's RI by directing subsidence warming into the developing upper-level warm core.

Hurricane Joaquin (2015) differed markedly from Wilma (2005). Its unusual looping track was poorly forecast by most operational models (Berg 2016; Doyle et al. 2017). The storm began a 60-h RI period at 0600 UTC 29 September, with its $V_{\text {MAX }}$ increasing from 18 to $61 \mathrm{~m} \mathrm{~s}^{-1}$. High SSTs exceeding $29^{\circ} \mathrm{C}$ appear to have facilitated the RI, despite moderate northerly VWS. Microwave satellite observations showed an asymmetric inner-core structure throughout Joaquin's early to middle RI period. Following RI, Joaquin slowly moved westward through the Bahamas, maintaining its intensity. Around 1800 UTC 2 October, soon after beginning its acceleration to the northeast, Joaquin began an 18-h reintensification, with its $V_{\mathrm{MAX}}$ increasing from 57 to $69 \mathrm{~m} \mathrm{~s}^{-1}$. Thereafter the storm weakened as it encountered lowering SSTs and increasing VWS. Miller and Zhang (2019) simulated Joaquin with WRF-ARW using a 27/9/3/1-km two-way interactive grid, 55 vertical $\sigma$ levels, and a $30-\mathrm{hPa}$ model top. The innermost domain, with $(x, y)$ dimensions of $601 \times 601$, followed the vortex center. WRF is initialized at RI onset with initial conditions generated from cycling hybrid data assimilation, and the outer three domains are nudged with a large-scale analysis. Results show that the model reproduced the RI rate and asymmetric inner-core structures reasonably well.

\section{b. General procedures for trajectory computations}

Aside from the AC algorithms, basic numerical elements of our trajectory calculations follow previous studies (Cram et al. 2007; Dahl et al. 2012; S15). The 1-km WRF moving nest from the Wilma (2005) and Joaquin (2015) simulations supplies the input data for all numerically simulated data trajectories. ARWpost ${ }^{2}$ software is used for destaggering and vertically interpolating WRF-output winds and desired scalars from their native Mercator projection $\mathrm{C}$ grid to a computational A grid, where scalars and all velocity components are defined at the vertices. The computational grid has a constant horizontal grid cell length $\Delta x=\Delta y=1000 \mathrm{~m}$ and a vertical resolution $\Delta z$ set to $250-\mathrm{m}(50 \mathrm{~m})$ above (below) $z=1 \mathrm{~km}$. Model-output 10-m horizontal winds and $w=0$ populate the ground level. Lateral boundaries

\footnotetext{
${ }^{2}$ Documentation is for the ARWpost postprocessing package is available at http://www2.mmm.ucar.edu/wrf/users/docs/user_guide_ V3.9/users_guide_chap9.htm\#_ARWpost_3.
} 
are set to the edges of the destaggered WRF grid, and the top boundary is set to $20 \mathrm{~km}$. Map-scale factors $m_{x}$ and $m_{y}$ relate $\Delta x$ and $\Delta y$ to their corresponding distances on the spherical Earth surface (Skamarock et al. 2008):

$$
\begin{aligned}
& m_{x}=\frac{\Delta x}{R_{e} \cos (\phi)|\Delta \lambda|} \\
& m_{y}=\frac{\Delta y}{R_{e} \Delta \phi},
\end{aligned}
$$

where $\phi$ and $\lambda$ are the latitude and longitude in radians, respectively; and $R_{e}$ is the mean radius of Earth. To correct for map distortion effects, the WRF-output $u$ and $v$ winds are transformed to the grid-relative winds:

$$
\begin{aligned}
& u_{\text {grid }}=u\left(m_{x}\right) \\
& v_{\text {grid }}=v\left(m_{y}\right) .
\end{aligned}
$$

Equation (1) is integrated using a second order RungeKutta (RK2) scheme (Press et al. 1992) with a 10-s computational time step. The parcel velocity at computational times is estimated from gridded winds at the nearest input times using either LI or AC in time and trilinear interpolation in space. Any parcel arriving at the grid top or lateral boundaries is flagged as having left the domain and its trajectory integration is terminated.

For the two numerically simulated data cases, an additional complication arises from the fact that the WRF vortex-following nest translates horizontally by several grid points between some output times. Prior to trajectory runtime, the grid translation vectors for the $x$ and $y$ directions, $x_{\mathrm{TRANS}}$ and $y_{\mathrm{TRANS}}$, respectively, are determined for every output time interval using the WRFoutput latitude and longitude and map-scale factors ${ }^{3}$; for output intervals where the domain remains stationary, $x_{\text {TRANS }}$ and $y_{\text {TRANS }}$ are set to zero. These vectors have units of (number of grid points) $\times \Delta x$, with a positive (negative) sign for northward and eastward (southward and westward) domain shifts, in the time direction of model integration. Trajectory calculations are kept in an Earth-relative framework by adding a correction term to Eq. (1) that opposes the effect of domain translation on parcel movement. For the $i$ th RK2 iteration, equations for parcel displacement in the respective $x$ and $y$ directions, $\delta x_{i}$ and $\delta y_{i}$, are

\footnotetext{
${ }^{3}$ For both the Wilma (2005) and Joaquin (2015) trajectory integration periods, the $1-\mathrm{km}$ domain moved in increments of 3 grid cells in one or both horizontal directions. This distance corresponds to 1 grid length in the parent nest.
}

$$
\begin{gathered}
\delta x_{i}=u_{\text {grid }, i} \delta t-x_{\text {TRANS }} \frac{\delta t}{\Delta t}, \\
\delta y_{i}=v_{\text {grid }, i} \delta t-y_{\text {TRANS }} \frac{\delta t}{\Delta t},
\end{gathered}
$$

where $u_{\text {grid }, i}\left(v_{\text {grid }, i}\right)$ are the local $u$ winds ( $v$ winds), the computational time step $\delta t$ is positive (negative) for forward (backward) trajectories, and $\Delta t$ is the model output time interval.

\section{c. Advection correction overview}

AC of model-output winds and scalars is performed in the horizontal plane, one vertical level at a time. Our rationale for defining the horizontal advective flows $U_{r}$ and $\Omega$ in cylindrical coordinates is the fact that on the vortex scale, TCs are often analyzed in terms of a swirling quasi-axisymmetric horizontal wind or "primary circulation," and a "secondary circulation" consisting of a low-level inflow, eyewall updraft core, and upper-level outflow (Liu et al. 1999; Montgomery and Smith 2014). Appendix A describes the methods used to determine the vortex center, and Fig. 2a summarizes the AC algorithm used for numerically simulated data cases; modifications for the analytical tests will be noted in section 3. Prior to running trajectories, the computational grid is divided into nonoverlapping horizontal subdomains, and $U_{r}$ and $\Omega$ are computed for each subdomain, one level at a time, for all model output time intervals $\Delta t$ using either the procedure described in section $2 \mathrm{~d}$ or as subdomain-averaged $u_{r}$ and $\omega$. Each level is divided into concentric annuli that extend outward from the storm center to a specified outer radial boundary; these annuli are further subdivided into arcs. Similar subdomain configurations are used for the two hurricane cases. For Wilma (Joaquin), 2-km (3-km) wide annuli are subdivided into $24^{\circ}$ wide arcs over an AC region extending out to a $60-\mathrm{km}(180-\mathrm{km})$ radius beyond the azimuthal mean radius of maximum wind (RMW) over the $z=0-16-\mathrm{km}(z=0-10 \mathrm{~km})$ layer. Standard LI is used in lieu of AC outside of this region.

During trajectory integrations, 3D arrays of Cartesian velocity vector components $u, v, w$ and desired scalars are generated at every computational time by temporally interpolating model data from the nearest output times. Virtual particles (borrowing S15's terminology) are launched backward and forward in time ${ }^{4}$ with velocity $\left(-U_{r},-\Omega\right)$ and $\left(U_{r}, \Omega\right)$, respectively, from every grid point $(x, y, z)$ within the AC subdomain

\footnotetext{
${ }^{4}$ Here "time" refers to real, or simulation time, which of course is opposite to the time direction for integrating backward trajectories.
} 
(a)

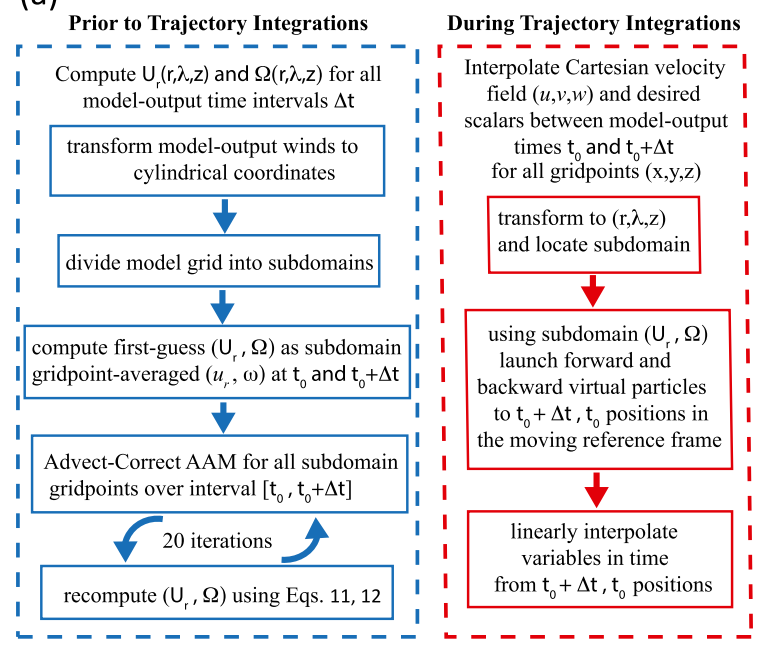

(b)

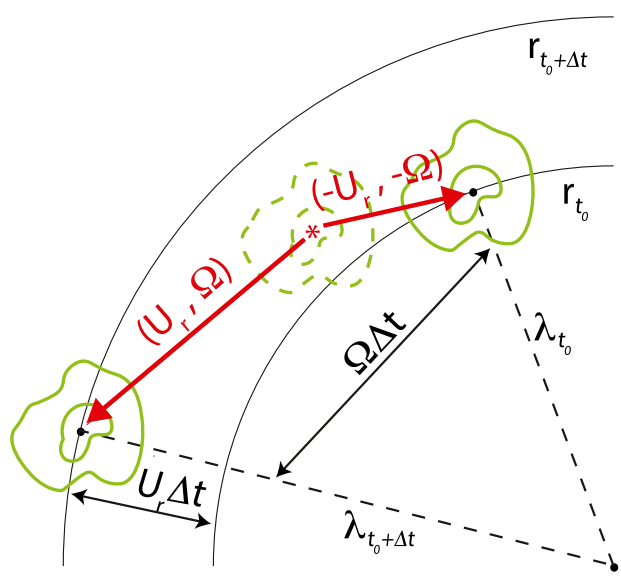

FIG. 2. (a) Block diagram outlining the key steps of the advection correction algorithm: the left column describes computation of the subdomain advective flows $\Omega$ and $U_{r}$, while the right column shows how $\Omega$ and $U_{r}$ are used to compute trajectories (see text). (b) Schematic illustrating advection correction in a cylindrical coordinate framework. An updraft element (solid green contours) translates counterclockwise with angular velocity $\Omega$ and radially outward with linear velocity $U_{r}$ between times $t=t_{0}$ and $t=t_{0}+\Delta t$. The updraft amplitude at an arbitrary intermediate time (dashed green contours) can be estimated by launching virtual particles (red arrows) to the updraft's coordinates $(r, \lambda)$ at times $t=t_{0}$ and $t=t_{0}+\Delta t$ and then linearly interpolating data in time from these two positions.

configuration. They are free to leave their subdomains, and their "landing positions" are the advectioncorrected coordinates of $(x, y, z)$ at the nearest model output times (Fig. 2b). Model output data are bilinearly interpolated in space to the landing positions; data from the landing positions are subsequently linearly interpolated in time to yield values for $(x, y, z)$ at the computational time. How reasonable, one may ask, is the assumption that perturbations in 3D flows and thermodynamic variables all maintain their amplitudes as they are advected by a mean flow pattern? For a given trajectory, are there cases where AC of $w$ improves accuracy, but AC of $u$ and $v$ has negative impacts, and vice versa? We shall revisit these questions in sections 4 and 5 when we compare numerically simulated data trajectories run using $\mathrm{AC}$ of the full Cartesian vector $(u, v, w)$ versus the $w$ component only.

\section{d. Solving for the subdomain advective flows $U_{r}$ and $\Omega$}

Our iterative method for obtaining $U_{r}$ and $\Omega$ on each subdomain closely follows the "iterative GalChen-based procedure" used by S15 to solve for their $U$ and $V$, but with the following modifications: (i) applying the frozen turbulence constraint to AAM instead of the $u$ field, (ii) tracking the pattern advection in cylindrical rather than Cartesian coordinates, and (iii) adding a vertical advection constraint on the solution. Let us define a cost function $J$ that penalizes deviations from AAM conservation following the flow (i.e., the condition of $D \mathrm{AAM} / D t=0)$ :

$$
\begin{aligned}
J\left(U_{r}, \Omega\right)= & \iiint_{\text {subdomain }, \Delta t}\left(\frac{\partial \mathrm{AAM}}{\partial t}+\bar{w} \frac{\overline{\partial \mathrm{AAM}}}{\partial z}\right. \\
& \left.+U_{r} \frac{\partial \mathrm{AAM}}{\partial r}+\Omega \frac{\partial \mathrm{AAM}}{\partial \lambda}\right)^{2} r d r d \lambda d t,
\end{aligned}
$$

where the integration extends over all subdomain grid points and computational times within the model output interval $\Delta t . U_{r}$ and $\Omega$ are treated as constants over each subdomain. Here vertical AAM advection is not explicitly solved for but instead treated as a constant penalty term, parameterized as $\bar{w}(\overline{\partial \mathrm{AAM} / \partial z})$, with overbars denoting subdomain gridpoint averages. Horizontal advections $U_{r}$ and $\Omega$ that minimize $J$ can be found by setting $\partial J / \partial U_{r}=0$ and $\partial J / \partial \Omega=0$ to yield the following system of equations:

$$
\begin{aligned}
& A U_{r}+B \Omega=D \\
& B U_{r}+C \Omega=E,
\end{aligned}
$$

where 


$$
\begin{aligned}
A= & \iiint_{\text {subdomain }, \Delta t}\left(\frac{\partial \mathrm{AAM}}{\partial r}\right)^{2} r d r d \lambda d t \\
B= & \iiint_{\text {subdomain }, \Delta t}\left(\frac{\partial \mathrm{AAM}}{\partial r}\right)\left(\frac{\partial \mathrm{AAM}}{\partial \lambda}\right) r d r d \lambda d t \\
C= & \iiint_{\text {subdomain }, \Delta t}\left(\frac{\partial \mathrm{AAM}}{\partial \lambda}\right)^{2} r d r d \lambda d t \\
D= & -\iiint_{\text {subdomain, } \Delta t}\left(\frac{\partial \mathrm{AAM}}{\partial t}+\bar{w} \frac{\overline{\partial A \mathrm{AM}}}{\partial z}\right) \\
& \times\left(\frac{\partial \mathrm{AAM}}{\partial r}\right) r d r d \lambda d t \\
E= & -\iiint_{\text {subdomain, } \Delta t}\left(\frac{\partial \mathrm{AAM}}{\partial t}+\bar{w} \frac{\overline{\partial \mathrm{AAM}}}{\partial z}\right) \\
& \times\left(\frac{\partial \mathrm{AAM}}{\partial \lambda}\right) r d r d \lambda d t
\end{aligned}
$$

The equations for $U_{r}$ and $\Omega$ then become

$$
U_{r}=\frac{C D-E B}{A C-B^{2}} \quad \text { and } \quad \Omega=\frac{A E-B D}{A C-B^{2}} .
$$

Taking the second derivatives $\partial^{2} J / \partial U_{r}^{2}, \partial^{2} J / \partial \Omega^{2}$, and $\partial^{2} J / \partial U_{r} \partial \Omega$, and invoking the second derivative test (Stewart 1999, p. 974) with the Cauchy-SchwarzBuniakowski inequality for integrals (Gradshteyn and Ryzhik 2007, p. 1064) reveals that the solution corresponds to a minimum (rather than a maximum) in $J$.

Following GC82 and S15, we minimize $J$ iteratively. The procedure is initialized with "first-guess" advections, which are set to the subdomain-averaged $u_{r}$ and $\omega$. They define the velocities of the virtual particles used for temporally interpolating the model-output AAM field to all computational timesteps spanning $\Delta t$, as described in the preceding subsection. Integrals $A-E$ in Eq. (11) are then computed from the advection-corrected AAM field, and $U_{r}$ and $\Omega$ are updated using Eq. (12). This cycle is run for 20 iterations, and the code is flagged for instances of nonconvergence, defined here as either $\Delta U_{r} \geq 0.1 \mathrm{~m} \mathrm{~s}^{-1}$ or $\Delta \Omega \geq 0.001^{\circ} \mathrm{s}^{-1}$ between the 19th and 20th iterations. We forego this iterative procedure for the boundary layer $(z<1 \mathrm{~km})$ subdomains where large departures from AAM material conservation can be expected due to friction. Here, the advective flows are set to the subdomainaveraged first guesses, and they are blended with the $z=1 \mathrm{~km} \mathrm{Gal}-$ Chen advections over the upper portion.

Unfortunately, solution uniqueness for $U_{r}$ and $\Omega$ cannot be guaranteed. Shapiro et al. (2010) and S15 showed how this problem is inherent to any Gal-Chenbased procedure used in determining advective flows from scalar fields available at discrete input times. Although the partial derivatives $\partial \mathrm{AAM} / \partial t, \partial \mathrm{AAM} / \partial r$, and $\partial \mathrm{AAM} / \partial \lambda$ are treated as constants when differentiating Eq. (9) with respect to $U_{r}$ and $\Omega$, they are actually implicit functions of the advective flows, causing Eqs. (10a) and (10b) to become nonlinear. Shapiro et al. (2010) showed analytically how an infinite family of $U$ solutions could be retrieved by a Gal-Chen-based procedure for a wave pattern translating with constant velocity $U_{\text {true }}$, with the nearest spurious solutions moving closer to $U_{\text {true }}$ as the data input time interval increases. S15 found a similar result in their numerically simulated data tests, although they also found that choosing reasonable first-guess $U$ and $V$ values reduced the likelihood of convergence to spurious solutions. Solution nonuniqueness will be explored herein in sections 3 and 4 .

\section{e. Calculation of trajectory errors}

Trajectory errors are quantified in terms of position differences from LI "reference trajectories" seeded from the same locations and model output times and run using higher-temporal resolution input data. Horizontal and vertical position errors are defined separately due to the fact that $\mathrm{TC}$ vertical length scales are $\sim O(10) \mathrm{km}$ smaller than horizontal length scales. Borrowing the S15 notation, the horizontal individual dis-

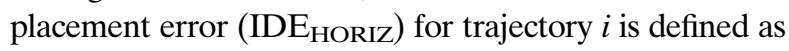

$$
\mathrm{IDE}_{\mathrm{HORIZ,EXP}}^{i}=\sqrt{\left(x_{\mathrm{EXP}}^{i}-x_{R}^{i}\right)^{2}+\left(y_{\mathrm{EXP}}^{i}-y_{R}^{i}\right)^{2}},
$$

where endpoint position $(x, y)$ is evaluated at a specified integration time and subscripts EXP and $R$ denote the experiment, either LI or AC, and reference trajectory, respectively. Likewise, we may define a vertical individual displacement error (IDE VERT $_{\text {): }}$

$$
\mathrm{IDE}_{\mathrm{VERT}, \mathrm{EXP}}^{i}=\left|z_{\mathrm{EXP}}^{i}-z_{R}^{i}\right|
$$

at endpoint position $z$. The impacts of AC are measured in terms of the change in horizontal and vertical position errors when a sample of LI trajectories is rerun from the same temporal input resolution using AC. Differenced horizontal individual displacement errors $\left(\Delta \mathrm{IDE}_{\mathrm{HORIZ}}\right)$ and vertical individual displacement errors $\left(\Delta\right.$ IDE $\left._{\text {VERT }}\right)$ are defined for trajectory $i$ as

$$
\begin{gathered}
\Delta \mathrm{IDE}_{\mathrm{HORIZ}}^{i}=\mathrm{IDE}_{\mathrm{HORIZ}, \mathrm{LI}}^{i}-\mathrm{IDE}_{\mathrm{HORIZ}, \mathrm{AC}}^{i} \\
\Delta \mathrm{IDE}_{\mathrm{VERT}}^{i}=\mathrm{IDE}_{\mathrm{VERT}, \mathrm{LI}}^{i}-\mathrm{IDE}_{\mathrm{VERT}, \mathrm{AC}}^{i} .
\end{gathered}
$$

Therefore, a positive (negative) $\Delta \mathrm{IDE}_{\mathrm{HORIZ}}$ indicates reduced (increased) horizontal position errors for trajectory $i$, relative to the reference trajectory, when $\mathrm{AC}$ is used in lieu of LI; likewise for $\triangle \mathrm{IDE}_{\mathrm{VERT}}$ and vertical position errors. 

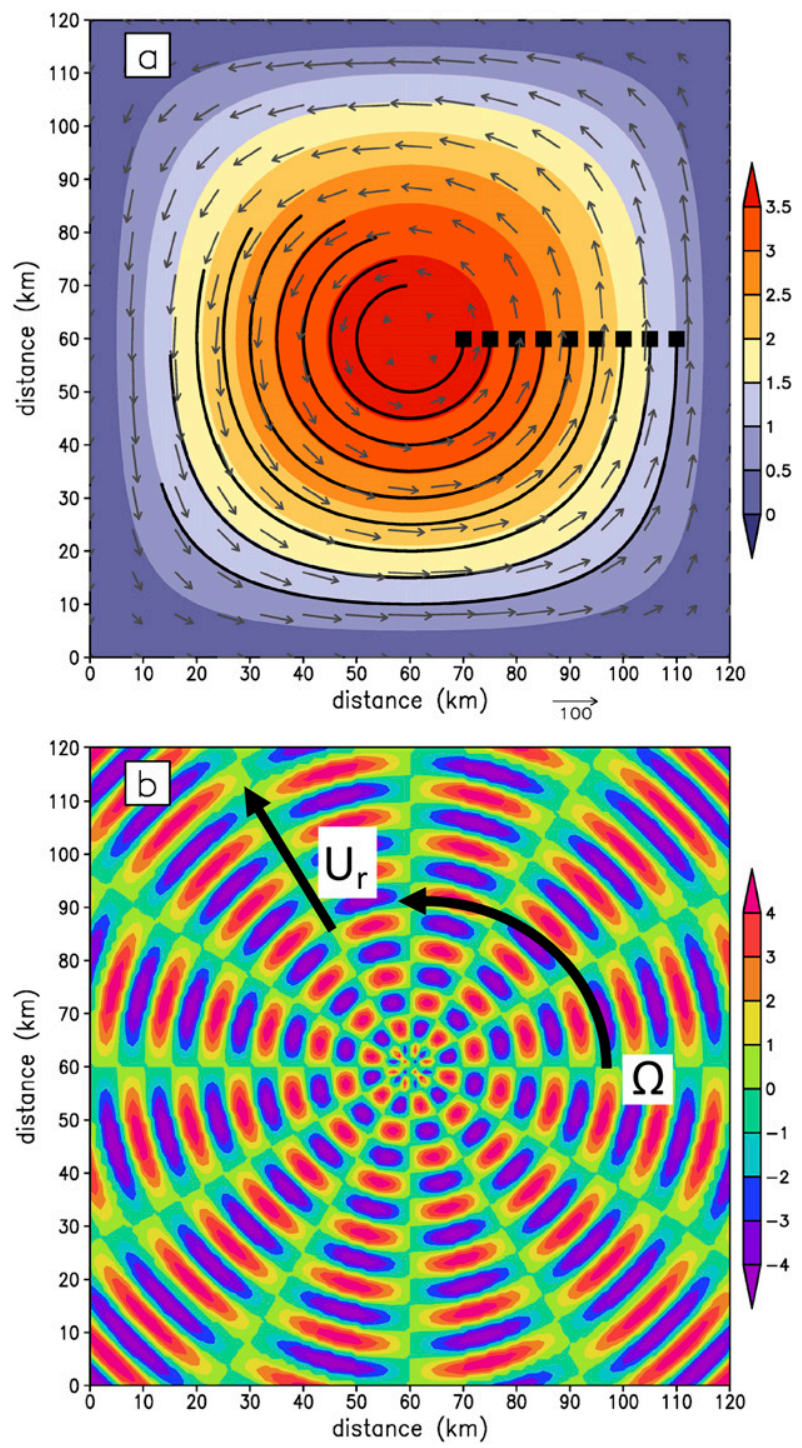

FIG. 3. (a) Horizontal flow field used for the analytical tests, with streamfunction (shaded, $10^{-3} \mathrm{~m}^{2} \mathrm{~s}^{-1}$ ) and flow vectors $\left(\mathrm{m} \mathrm{s}^{-1}\right)$. Representative 30-min backward trajectories for the first analytical test are shown in black, with squares denoting their seeded initial positions. (b) Horizontal variation of vertical motion at the initial time (shaded, $\mathrm{m} \mathrm{s}^{-1}$ ) used for the second analytical test. This vertical motion pattern translates azimuthally counterclockwise and radially outward over time, as shown by the black arrows.

\section{Analytical tests of trajectory computation}

Our first analytical test runs LI trajectories on a steadystate flow field in order to verify that the RK2 time integration and trilinear interpolation modules are functioning properly. The computational grids have $(x, y, z)$ dimensions of $121 \times 121 \times 41$, with horizontal and vertical lengths set to $\Delta x=\Delta y=1 \mathrm{~km}$ and $\Delta z=0.25 \mathrm{~km}$, respectively. Horizontal flows are invariant with height, and they are based on the Taylor-Green (1937) vortex initial conditions, in which

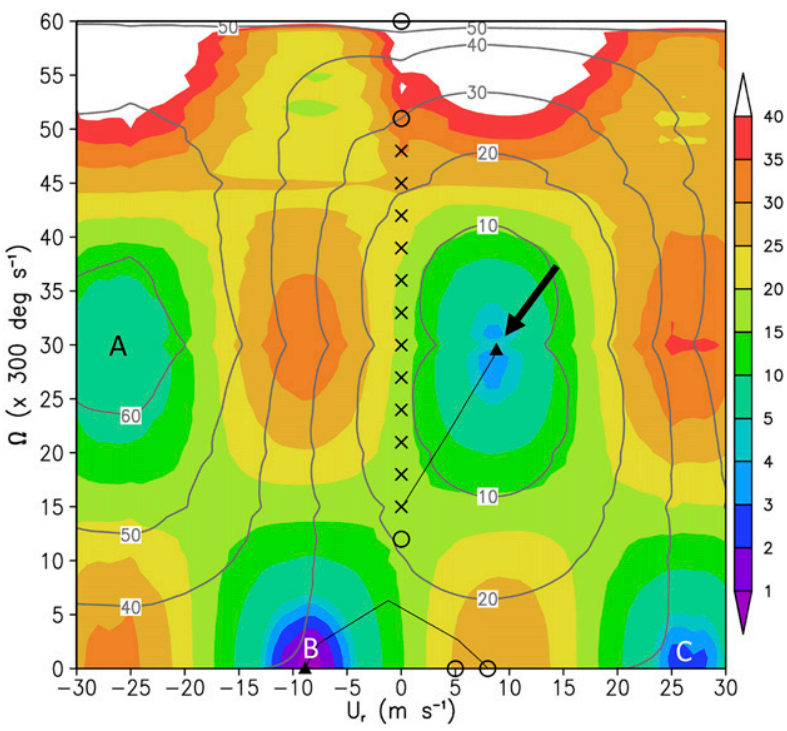

FIG. 4. Cost function $J\left(U_{r}, \Omega\right)\left(\right.$ shaded, $\left.\mathrm{m}^{4} \mathrm{~s}^{-3} \times 1.8 / \pi \times 10^{5}\right)$ evaluated for a selected subdomain on the analytical flow field shown in Fig. $3 \mathrm{~b}$ for the 5-min data input interval (shaded) and the 2.5-min data input interval (contours) ending at $t=30 \mathrm{~min}$. To compute $J$, Eq. (9) is solved using a range of input $U_{r}$ and $\Omega$ values, with AAM replaced by $w$. Black arrow points to the local minimum corresponding to the true advective flows (i.e., $U_{r}=8.33 \mathrm{~m} \mathrm{~s}^{-1}, \Omega=$ $\left.0.1^{\circ} \mathrm{s}^{-1}\right)$. Letters $\mathrm{A}, \mathrm{B}$, and $\mathrm{C}$ label spurious local minima (see text). Black $\times$ symbols (open circles) show first-guess $\left(U_{r}, \Omega\right)$ combinations that converged to values close to the true advective flows (converged to spurious solutions) using the iterative Gal-Chen procedure with 5 -min input data. Black line segments connect intermediate $\left(U_{r}, \Omega\right)$ solutions found over the 20 iterations leading to the final $\left(U_{r}, \Omega\right)$ solution, denoted by black triangles, for two selected first guesses.

$$
\begin{aligned}
& u(x, y)=V_{\text {max }} \sin \left(\frac{\pi x}{L}\right) \cos \left(\frac{\pi y}{L}\right), \\
& v(x, y)=-V_{\text {max }} \cos \left(\frac{\pi x}{L}\right) \sin \left(\frac{\pi y}{L}\right),
\end{aligned}
$$

where $V_{\max }=100 \mathrm{~m} \mathrm{~s}^{-1}$ and $L=120 \mathrm{~km}$. Horizontal components of this inviscid and incompressible flow field can be described by a streamfunction $\psi$, where $u=\partial \psi / \partial y$ and $v=-\partial \psi / \partial x$ :

$$
\psi(x, y)=\frac{V_{\max } L}{\pi} \sin \left(\frac{\pi x}{L}\right) \sin \left(\frac{\pi y}{L}\right) .
$$

The horizontally uniform vertical velocity field is defined by $w(z)=k z$, with $k=0.001 \mathrm{~s}^{-1}$. A batch of 984 backward trajectories is seeded in a concentric circular pattern ${ }^{5}$ from the $z=9-\mathrm{km}$ level and run for $30 \mathrm{~min}$, and selected horizontal projections are shown in Fig. 3a, superimposed upon the horizontal flow field. Since the

\footnotetext{
${ }^{5}$ Trajectories are seeded at $15^{\circ}$ azimuthal intervals for every radius between $r=10 \mathrm{~km}$ and $r=50 \mathrm{~km}$.
} 

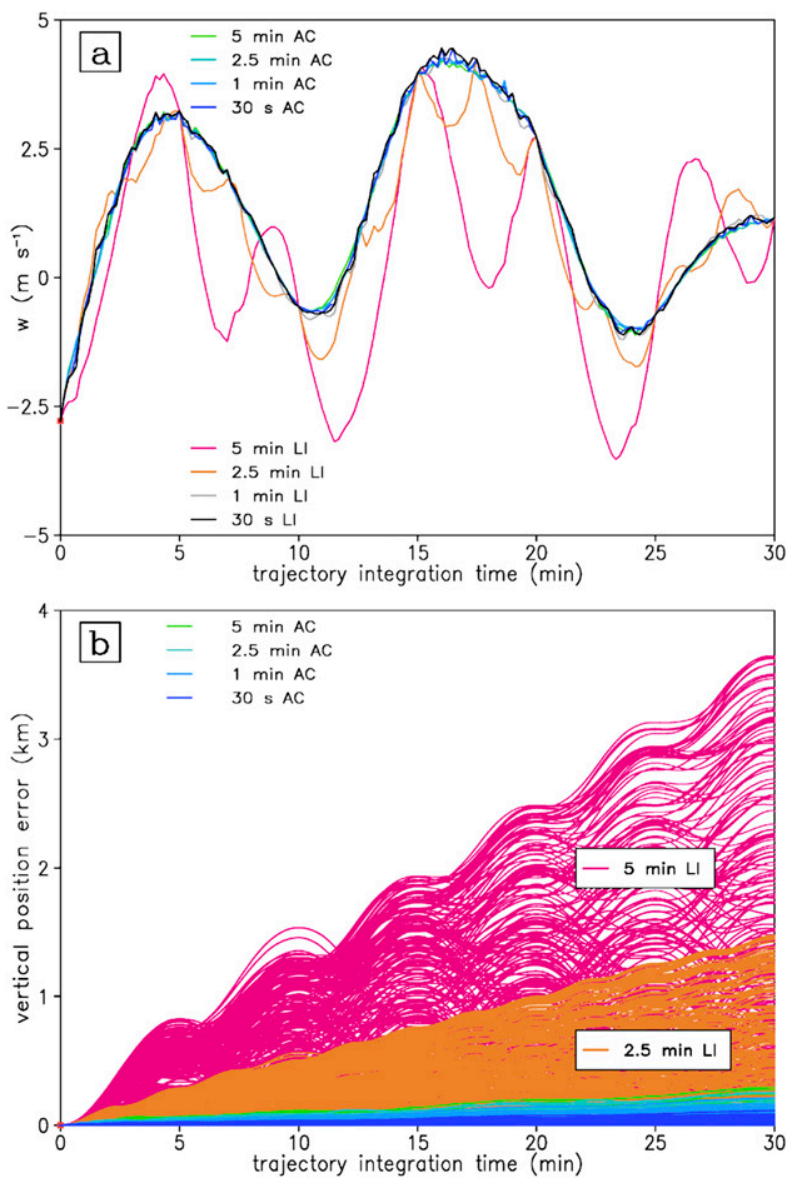

FIG. 5. Results of the second analytical test (see text) showing (a) vertical velocity along a representative backward trajectory, with linear interpolation (LI) and advection correction (AC) time interpolation methods both tested for 5-min, 2.5-min, 1-min, and 30-s data input times and (b) individual height displacement errors as a function of time along all 984 backward trajectories from the experiments shown in (a). The 1-min LI trajectory error growth pattern (not shown) closely resembles that of the 5-min AC trajectories. Reference trajectories are computed from the 30-s input data using LI.

horizontal winds are steady state, trajectories should follow contours of $\psi$. Visual inspection of Fig. 3a suggests that trajectories are closely following streamlines, and deviations of $\psi$ from its initial value along all trajectories run are found to be less than $0.02 \%$ (not shown). Backward trajectory height-versus-time traces follow the function $z=z_{0} e^{-k t}$ with $z_{0}=9 \mathrm{~km}$ (not shown)-this is the exact solution to the differential equation for parcel height along all backward trajectories.

The second test is designed to verify that the $\mathrm{AC}$ algorithms are free of code errors. It uses the same computational grid and horizontal flows used for the first test, but with the steady-state $w=k z$ field replaced with a horizontally varying pattern (Fig. $3 b$ ) that rotates cyclonically and translates radially outward. We keep
TABLE 1. Advection correction experiments.

\begin{tabular}{lcl}
\hline \hline \multicolumn{1}{c}{ Name } & $\begin{array}{c}\text { Wind vector } \\
\text { components } \\
\text { interpolated }\end{array}$ & \multicolumn{1}{c}{$\begin{array}{c}\text { Procedure for } \\
\text { finding } U_{r} \text { and } \Omega\end{array}$} \\
\hline $\mathrm{AC}_{\mathrm{W}}$ & $w$ & Iterative Gal-Chen \\
$\mathrm{AC}_{\mathrm{UVW}}$ & $u, v, w$ & Iterative Gal-Chen \\
$\mathrm{AC}_{\mathrm{MW}}$ & $u, v, w$ & Use subdomain-averaged flows \\
\hline
\end{tabular}

$w$ constant with height. In cylindrical coordinates, it can be expressed as

$w(r, \lambda, t)=w_{\max } \sin \left(2 \pi \frac{\lambda-\Omega t}{L_{\lambda}}\right) \sin \left(2 \pi \frac{r-U_{r} t}{L_{r}}\right)$,

with azimuthal angle $\lambda$ measured counterclockwise from the positive $x$ axis, radius $r$ measured from the domain center, and parameters $w_{\max }=5 \mathrm{~m} \mathrm{~s}^{-1}, L_{\lambda}=60^{\circ}, L_{r}=$ $10000 \mathrm{~m}, \Omega=0.1^{\circ} \mathrm{s}^{-1}$, and $U_{r}=8.33 \mathrm{~m} \mathrm{~s}^{-1}$. Using the product rule, Eq. (20) can be shown to satisfy $\partial w / \partial t+$ $U_{r}(\partial w / \partial r)+\Omega(\partial w / \partial \lambda)=0$; thus, $w$ is conserved for a parcel following the advection flow vector $\left(U_{r}, \Omega\right)$. The 984 30-min backward trajectories from the first test are relaunched from $z=5 \mathrm{~km}$, using both $\mathrm{LI}$ and $\mathrm{AC}$, on flows with varying temporal resolution $\Delta t: 5 \mathrm{~min}, 2.5 \mathrm{~min}$, $1 \mathrm{~min}$, and $30 \mathrm{~s}$. The 30 -s LI experiment supplies the "reference trajectories." AC is applied to the $w$ field only. For AC experiments, each level is divided into subdomain arcs with $(r, \lambda)$ resolutions of $4 \mathrm{~km} \times 30^{\circ}$. Since $w$ is conserved following the advective flow by design, we replace AAM in Eqs. (9) and (11) with $w$ when solving for $U_{r}$ and $\Omega$. The AC algorithm otherwise follows the one described in sections $2 \mathrm{c}$ and $2 \mathrm{~d}$.

Before examining trajectory errors, let us first verify that the iterative Gal-Chen-based procedure can converge upon the true $\Omega$ and $U_{r}$, given different first-guess values. Figure 4 shows the cost function $J\left(U_{r}, \Omega\right)$ computed by "brute force" for a selected subdomain from the 5-min output data for a range of $U_{r}$ and $\Omega$ inputs. For each $U_{r}$ and $\Omega$ pair, the subdomain $w$ field is advectioncorrected over a $\Delta t=5$-min period using a 10 -s computational time step and then Eq. (9) is evaluated. As expected, a local minimum can be found for the "true" advections $\left(U_{r}=8.33 \mathrm{~m} \mathrm{~s}^{-1}, \Omega=0.1^{\circ} \mathrm{s}^{-1}\right)$. Note, however, the regularly spaced pattern of additional local minima A, B, and C located at $\left(U_{r} \sim-25.0 \mathrm{~m} \mathrm{~s}^{-1}, \Omega \sim\right.$ $\left.0.1^{\circ} \mathrm{s}^{-1}\right),\left(U_{r} \sim-9.0 \mathrm{~m} \mathrm{~s}^{-1}, \Omega \sim 0.0^{\circ} \mathrm{s}^{-1}\right)$, and $\left(U_{r} \sim\right.$ $\left.26.0 \mathrm{~m} \mathrm{~s}^{-1}, \Omega \sim 0.0^{\circ} \mathrm{s}^{-1}\right)$, respectively; this is consistent with $J$ being nonunique in $U_{r}$ and $\Omega$, as discussed in section $2 \mathrm{~d}$. To understand the physical basis for these spurious minima, consider $w(r, \lambda, t)$ at two data input times $t=0$ and $t=T$, denoted by $w_{0}$ and $w_{T}$, respectively: 

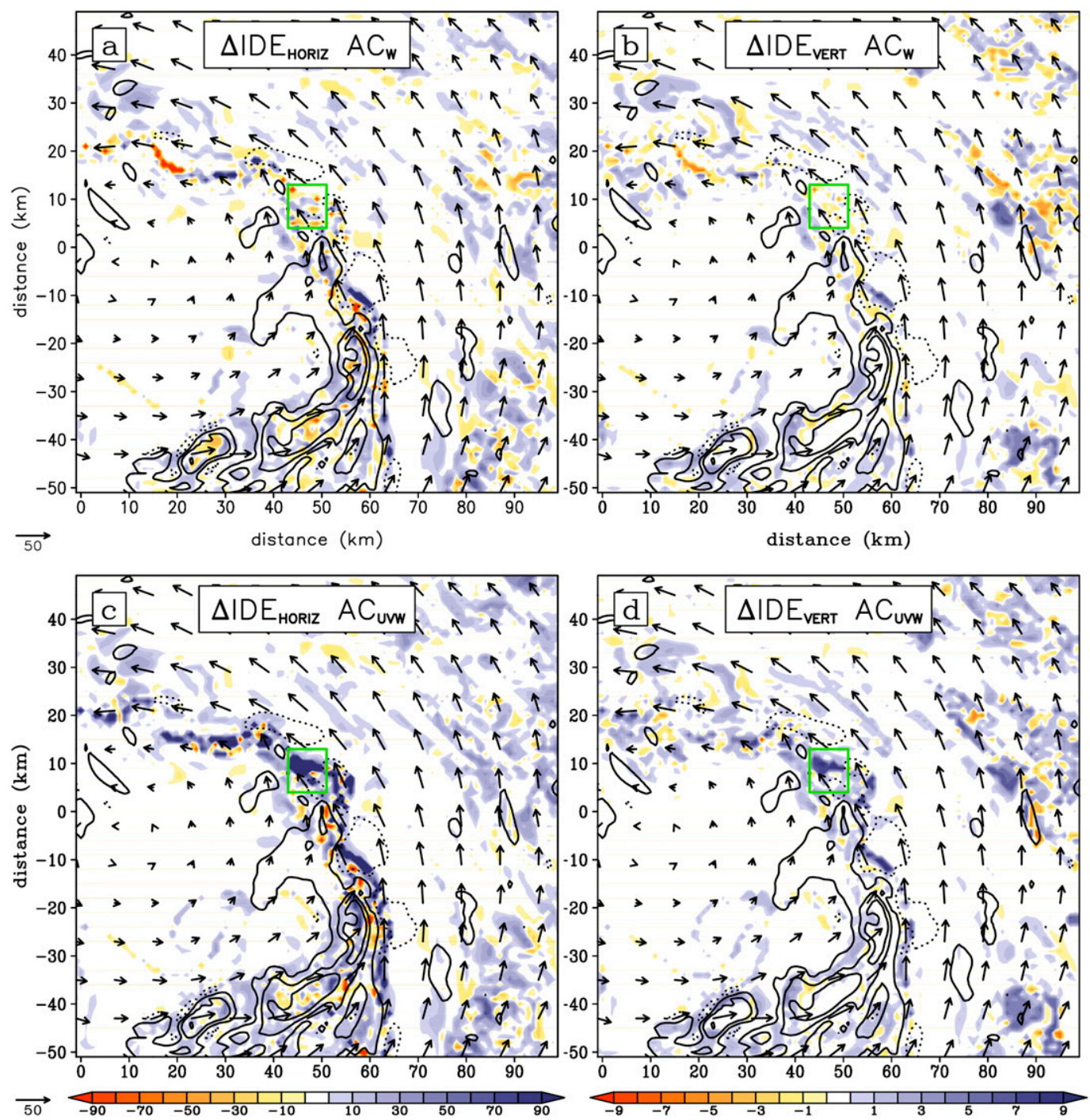

FIG. 6. Distribution of vertical velocity (black solid contours at $1 / 5 / 10 \mathrm{~m} \mathrm{~s}^{-1}$; black dotted contours at $-2 \mathrm{~m} \mathrm{~s}^{-1}$ ) and horizontal storm-relative flow vectors $\left(\mathrm{m} \mathrm{s}^{-1}\right.$ ) taken from the WRF-simulated Hurricane Joaquin (2015) 1-km resolution domain at $z=6 \mathrm{~km}$ and $t=2800$. Four-hour backward trajectories are seeded from this time at every 1-km grid point, for a total of 10201 trajectories. (a) Differenced 2-h horizontal individual displacement errors ( $\left.\triangle \mathrm{IDE}_{\mathrm{HORIZ}}\right)$ (shaded, every $10 \mathrm{~km}$ except every $5 \mathrm{~km}$ for $\left|\Delta \mathrm{IDE}_{\mathrm{HORIZ}}\right|<10 \mathrm{~km}$ ) plotted as a function of seed position for $\mathrm{AC}_{\mathrm{W}}$. (b) As in (a), but for differenced vertical individual displacement errors $\left(\Delta \mathrm{IDE}_{\mathrm{VERT}}\right.$ ) (shaded, every $1 \mathrm{~km}$ except every $0.5 \mathrm{~km}$ for $\left|\Delta \mathrm{IDE}_{\mathrm{VERT}}\right|<1 \mathrm{~km}$ ). (c),(d) As in (a) and (b), respectively, but for the $\mathrm{AC}_{\mathrm{UVW}}$ experiment. Horizontal distances shown here and for all subsequent figures, unless otherwise noted, are measured in $\mathrm{km}$ from the domain center, with negative values for regions south and west of the domain center.

$$
\begin{aligned}
& w_{0}=w_{\max } \sin \left(2 \pi \frac{\lambda}{L_{\lambda}}\right) \sin \left(2 \pi \frac{r}{L_{r}}\right), \\
& w_{T}=w_{\max } \sin \left(2 \pi \frac{\lambda-\Omega_{\text {true }} T}{L_{\lambda}}\right) \sin \left(2 \pi \frac{r-U_{r, \text { true }} T}{L_{r}}\right),
\end{aligned}
$$

where $\Omega_{\text {true }}$ and $U_{r \text {,true }}$ represent the true advection velocities. Because of $w(r, \lambda, t)$ being doubly periodic in $r$ and $\lambda$ (Fig. 3b), Eqs. (21a) and (21b) can also be satisfied by any $w_{T}$ phase shifted by integer multiples of 1) one wavelength in either $r$ or $\lambda$ or 2) one-half wavelength in both $r$ and $\lambda$. Thus, $\Omega_{\text {true }}$ and $U_{r, \text { true }}$ may be replaced with two families of infinite solutions:

$$
\begin{array}{cc}
U_{r}=U_{r, \text { true }}+\frac{m_{1} L_{r}}{T}, & m_{1}=0, \pm 1,2,3, \ldots ; \\
\Omega & =\Omega_{\text {true }}+\frac{m_{2} L_{\lambda}}{T}, \quad m_{2}=0, \pm 1,2,3, \ldots
\end{array}
$$

and 


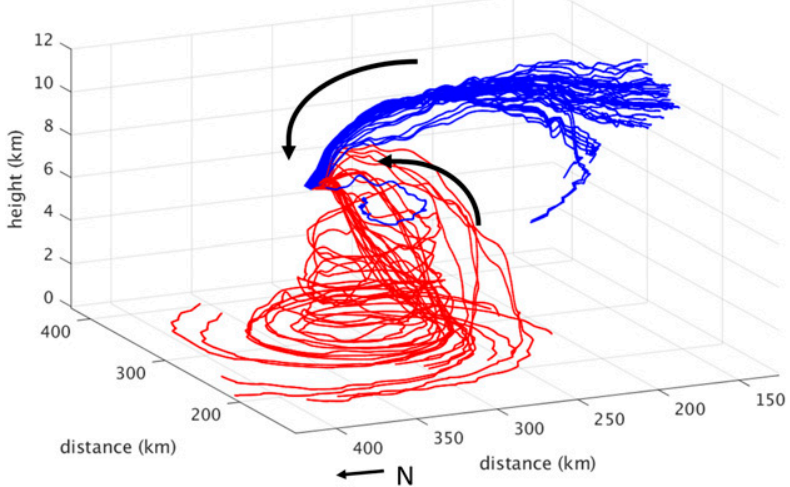

FIG. 7. Three-dimensional 4-h backward trajectories computed from the Hurricane Joaquin (2015) WRF simulation 5-min output using advection correction of the horizontal and vertical velocity fields $\left(\mathrm{AC}_{\mathrm{UVw}}\right)$. All trajectories shown here are seeded at $z=6 \mathrm{~km}$ forecast hour 2800, and they are selected from within the rectangular region shown in Fig. 6. They are stratified by final (i.e., forecast hour 2400) height, with red colors showing trajectories originating below $z=1 \mathrm{~km}$ (23 total) and blue colors showing trajectories originating from above $z=6 \mathrm{~km}$ (40 total). Black arrows highlight the convergent flowpaths of the two backward trajectory clusters. Horizontal distances are measured from the $1-\mathrm{km}$ domain southwest corner.

$$
\begin{aligned}
U_{r}=U_{r, \text { true }}+\frac{n_{1} L_{r}}{T}, & n_{1}= \pm \frac{1}{2}, \frac{3}{2}, \frac{5}{2}, \ldots ; \\
\Omega & =\Omega_{\text {true }}+\frac{n_{2} L_{\lambda}}{T}, \quad n_{2}= \pm n_{1} \pm 0,1,2, \ldots
\end{aligned}
$$

Local minima A, B, and C from Fig. 4 are close to the Eq. (22) $\left(m_{1}=-1 ; m_{2}=0\right)$, Eq. (23) $\left[n_{1}=-(1 / 2)\right.$; $\left.n_{2}=-(1 / 2)\right]$, and Eq. (23) $\left[n_{1}=+(1 / 2) ; n_{2}=-(1 / 2)\right]$ solutions, respectively, for $T=300 \mathrm{~s}$. Equations (22) and (23) show how the spurious solutions become more distant from $\left(U_{r, \text { true }}, \Omega_{\text {true }}\right)$ as the data input interval $T$ decreases. Thus, only the local minimum $\left(U_{r \text {,true }}, \Omega_{\text {true }}\right)$ remains over the input $\left(U_{r}, \Omega\right)$ range shown in Fig. 4, when $\Delta t$ is reduced to $2.5 \mathrm{~min}$. The iterative Gal-Chen solver initialized with a range of first-guess $\left(U_{r}, \Omega\right)$ pairs converges on $\left(U_{r \text {,true }}, \Omega_{\text {true }}\right)$ as long as the first guesses are closer to $\left(U_{r \text {,true }}, \Omega_{\text {true }}\right)$ than to any of the spurious nodes (Fig. 4).

Figure 5a shows traces of $w(r, \lambda, t)$ interpolated along a representative trajectory using LI and AC over a range of $\Delta t$. All AC experiments use the iterative GalChen procedure initialized with $U_{r}=0 \mathrm{~m} \mathrm{~s}^{-1}$ and $\Omega=$ $0.06^{\circ} \mathrm{s}^{-1}$. LI estimates move increasingly far from the reference trajectory trace within data input intervals as $\Delta t$ increases, with $w$ errors growing to as large as $3 \mathrm{~m} \mathrm{~s}^{-1}$ for $\Delta t=5 \mathrm{~min}$; AC estimates, on the other hand, remain close to the reference solution, even for coarser $\Delta t$. Figure $5 \mathrm{~b}$ compares the growth of $\mathrm{IDE}_{\mathrm{VERT}, \mathrm{LI}}^{i}$ and $\mathrm{IDE}_{\mathrm{VERT}, \mathrm{AC}}^{i}$ along all 984 backward trajectories for the different $\Delta t$ experiments. A substantial spread in IDE $_{\text {VERT,LI }}^{i}$ among the trajectories develops over time for the coarser data input intervals $(\Delta t=2.5$ and $5 \mathrm{~min})$, as indicated by orange and magenta lines in Fig. $5 b$; this reflects the fact that $w$, translating with tangential velocity $\Omega r$, becomes locally less stationary in time with increasing radius and hence LI becomes an increasingly poorer $w$-estimator for large radii. ${ }^{6}$ Applying AC in lieu of LI reduces the 2.5- and 5-min IDE ${ }_{\mathrm{VERT}, \mathrm{LI}}^{i}$ by up to an order of magnitude, as shown by green and blue colored lines in Fig. 5b.

Results of the analytical tests described above suggest that the major components of our trajectory model can function properly when applied to a simple flow field containing perturbations that are perfectly conserved while translating through space. In the next section, we must determine whether AC can reduce trajectory errors for realistic TC flows, given several potential pitfalls that may become more problematic with coarser input data resolutions. They include the validity of the frozen-turbulence hypothesis and the potential for the iterative Gal-Chen procedure to retrieve spurious $\left(U_{r}, \Omega\right)$ solutions.

\section{Hurricane Joaquin (2015) tests}

The Hurricane Joaquin (2015) WRF simulation is output at 5- and 1-min temporal resolutions for the forecast period 2400-2800 (hhmm denotes hours and minutes). During this period, the category 1 hurricane is undergoing RI while the inner-core radar reflectivity structure remains relatively asymmetric, with the strongest convection in the southeastern eyewall (see Figs. 5c,d in Miller and Zhang 2019). Each experiment runs 4-h backward trajectories from every grid point over a $100 \mathrm{~km} \times 100 \mathrm{~km}$ horizontal section of the inner core at $z=6-\mathrm{km}$ height and time 2800 (10201 total). Table 1 summarizes the three AC implementations run from 5-min output that are tested against LI from 5-min output (hereafter LI-5). Both $\mathrm{AC}_{\mathrm{W}}$ and $\mathrm{AC}_{\mathrm{UVw}}$ solve for $U_{r}$ and $\Omega$ using the iterative Gal-Chen-based procedure described in section $2 \mathrm{~d} ; \mathrm{AC}_{\mathrm{W}}$ advection-corrects only vertical velocity, using LI for horizontal winds, while $\mathrm{AC}_{\mathrm{UVw}}$ advection-corrects the full $3 \mathrm{D}$ velocity vector $(u, v, w)$. AC-MW $\mathrm{MVw}_{\mathrm{UVw}}$ modifies $\mathrm{AC}_{\mathrm{UVw}}$ by setting $U_{r}$ and $\Omega$ to the subdomain-averaged "first-guess" radial and angular velocities. Error statistics are computed

\footnotetext{
${ }^{6}$ Recall that the backward trajectories follow the steady-state horizontal traces shown in Fig. 3a. Therefore, each of them remains at a nearly constant radius, although they are seeded over a large radial range.
} 

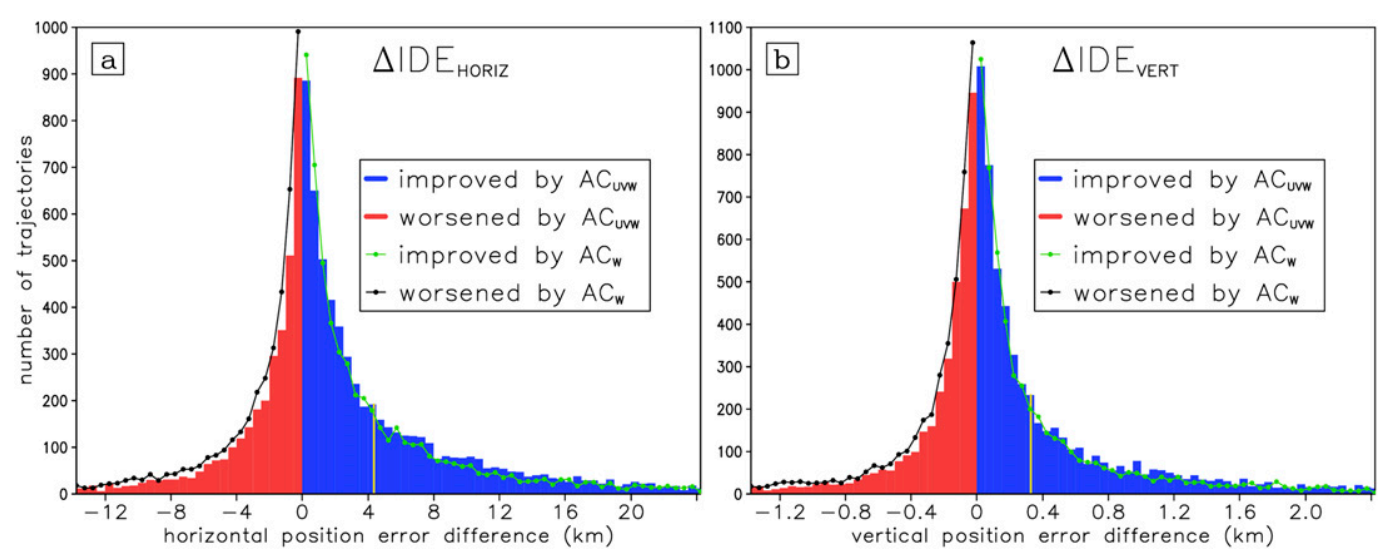

FIG. 8. (a) Histogram of differenced horizontal individual displacement errors $\left(\Delta \mathrm{IDE}_{\mathrm{HORIZ}}\right)$ for the $10201 \mathrm{AC}_{\mathrm{UVW}}$ trajectories, using a $0.25-\mathrm{km}$ bin width. Blue (red) bars show trajectories with improved (worsened) accuracy, as measured by a reduced (increased) $\mathrm{IDE}_{\mathrm{HORIZ}}$ compared to the same trajectories run from the 5-min model output using LI. Lines connect the histogram bin heights for the $10201 \mathrm{AC}_{\mathrm{W}}$ trajectories, with green (black) denoting improved (worsened) accuracy. (b) As in (a), but for $\triangle \mathrm{IDE}_{\text {VERT. }}$. Yellow vertical lines denote the sample mean values.

after $2 \mathrm{~h}$ of backward integration using reference LI trajectories run from the 1-min output data (hereafter LI-1).

\section{a. Trajectory error statistics}

Figure 6 plots $\Delta \mathrm{IDE}_{\mathrm{HORIZ}}$ and $\triangle \mathrm{IDE}_{\mathrm{VERT}}$ as a function of trajectory seed position for the $\mathrm{AC}_{\mathrm{W}}$ and $\mathrm{AC}_{\mathrm{UVW}}$ experiments. Note that $\triangle \mathrm{IDE}_{\mathrm{HORIZ}}$ and $\triangle \mathrm{IDE}_{\mathrm{VERT}}$ measure the change in horizontal and vertical 2-h position errors, respectively, for the $i$ th LI-5 trajectory when it is rerun using $\mathrm{AC}$, with positive (negative) values indicating improved (worsened) accuracy relative to the $i$ th reference LI-1 trajectory. As expected, replacing LI-5 with $\mathrm{AC}_{\mathrm{W}}$ primarily impacts trajectory accuracy in regions where the vertical motion field is highly variable in space (Figs. 6a,b) and nonstationary in time (not shown): namely, near and downstream of larger-scale ascent in the eastern eyewall and in portions of the outer circulation. The $\mathrm{AC}_{\mathrm{W}} \Delta \mathrm{IDE}_{\mathrm{HORIZ}}$ and $\triangle \mathrm{IDE}_{\mathrm{VERT}}$ patterns show some spatial correlation, which is not surprising, given that parcel vertical velocity corrections should lead to horizontal position corrections in this vertically sheared tangential wind circulation (not shown). Although $\mathrm{AC}_{\mathrm{W}}$ has some positive impact around the edges of the southeastern eyewall updraft core, it degrades a substantial percentage of the LI-5 trajectories seeded from the northeast eye-eyewall interface region. By contrast, $\mathrm{AC}_{\mathrm{UVw}}$ reduces LI-5 errors over a larger portion of the sample, as evident in the more widespread blue shading, and it performs particularly well in the inner northeast eyewall (Figs. 6c,d). For example, consider the boxed area where $\mathrm{AC}_{\mathrm{UVw}}$ reduces many LI-5 horizontal (vertical) trajectory errors by $>30 \mathrm{~km}(>3 \mathrm{~km})$. Many backward trajectories seeded in this box originate from the boundary layer, via eyewall ascent, and also from the outer environment, via a descending mid- to upper-level inflow channel (Fig. 7). Confluent flows present a challenging scenario for backward trajectory calculations, where relatively small uncertainties in the local wind estimation can lead to large position errors (Dahl et al. 2012).

These results are supported by histograms of $\Delta$ IDE $_{\text {HORIZ, given in Fig. }}$ 8a showing a rightward (positive) shift in the $A C_{U V W}$ distribution relative to $\mathrm{AC}_{\mathrm{W}}$. The $\triangle \mathrm{IDE}_{\mathrm{VERT}}$ distribution differs less between the two experiments; however, $\mathrm{AC}_{\mathrm{UVw}}$ overwhelmingly outperforms $\mathrm{AC}_{\mathrm{W}}$ for the extreme distribution tails $\left(\left|\Delta \mathrm{IDE}_{\mathrm{VERT}}\right|>1 \mathrm{~km}\right)$ not shown on these histograms

TABLE 2. Summary statistics for the $\mathrm{AC}_{\mathrm{W}}, \mathrm{AC}_{\mathrm{UVW}}$, and $\mathrm{AC}-\mathrm{MW}_{\mathrm{UVW}}$ experiments using the Hurricane Joaquin (2015) simulation dataset. Italicized values highlight the experiment with the most improved trajectory accuracy for each statistic.

\begin{tabular}{|c|c|c|c|c|c|c|}
\hline \multirow[b]{2}{*}{ Statistic } & \multicolumn{3}{|c|}{$\Delta \mathrm{IDE}_{\mathrm{HORIZ}}$} & \multicolumn{3}{|c|}{$\Delta \mathrm{IDE}_{\mathrm{VERT}}$} \\
\hline & $\mathrm{AC}_{\mathrm{W}}$ & $\mathrm{AC}_{\mathrm{UVW}}$ & AC-MW $W_{U V W}$ & $\mathrm{AC}_{\mathrm{W}}$ & $\mathrm{AC}_{\mathrm{UVW}}$ & AC-MW $W_{U V W}$ \\
\hline Mean $\Delta \mathrm{IDE}(\mathrm{km})$ & 1.42 & 4.44 & 4.97 & 0.11 & 0.33 & 0.39 \\
\hline$\%$ left tail ${ }^{9}$ & 5.3 & 4.0 & 3.8 & 5.4 & 3.6 & 3.6 \\
\hline$\%$ right tail ${ }^{10}$ & 10.1 & 15.5 & 16.2 & 8.7 & 13.1 & 13.9 \\
\hline
\end{tabular}

\footnotetext{
${ }^{9}$ Percentage of all trajectories with $\triangle \mathrm{IDE}_{\mathrm{HORIZ}}<-10 \mathrm{~km}, \Delta \mathrm{IDE}_{\mathrm{VERT}}<-1 \mathrm{~km}$.
}

${ }^{10}$ Percentage of all trajectories with $\Delta \mathrm{IDE}_{\mathrm{HORIZ}}>10 \mathrm{~km}, \Delta \mathrm{IDE}_{\mathrm{VERT}}>1 \mathrm{~km}$. 

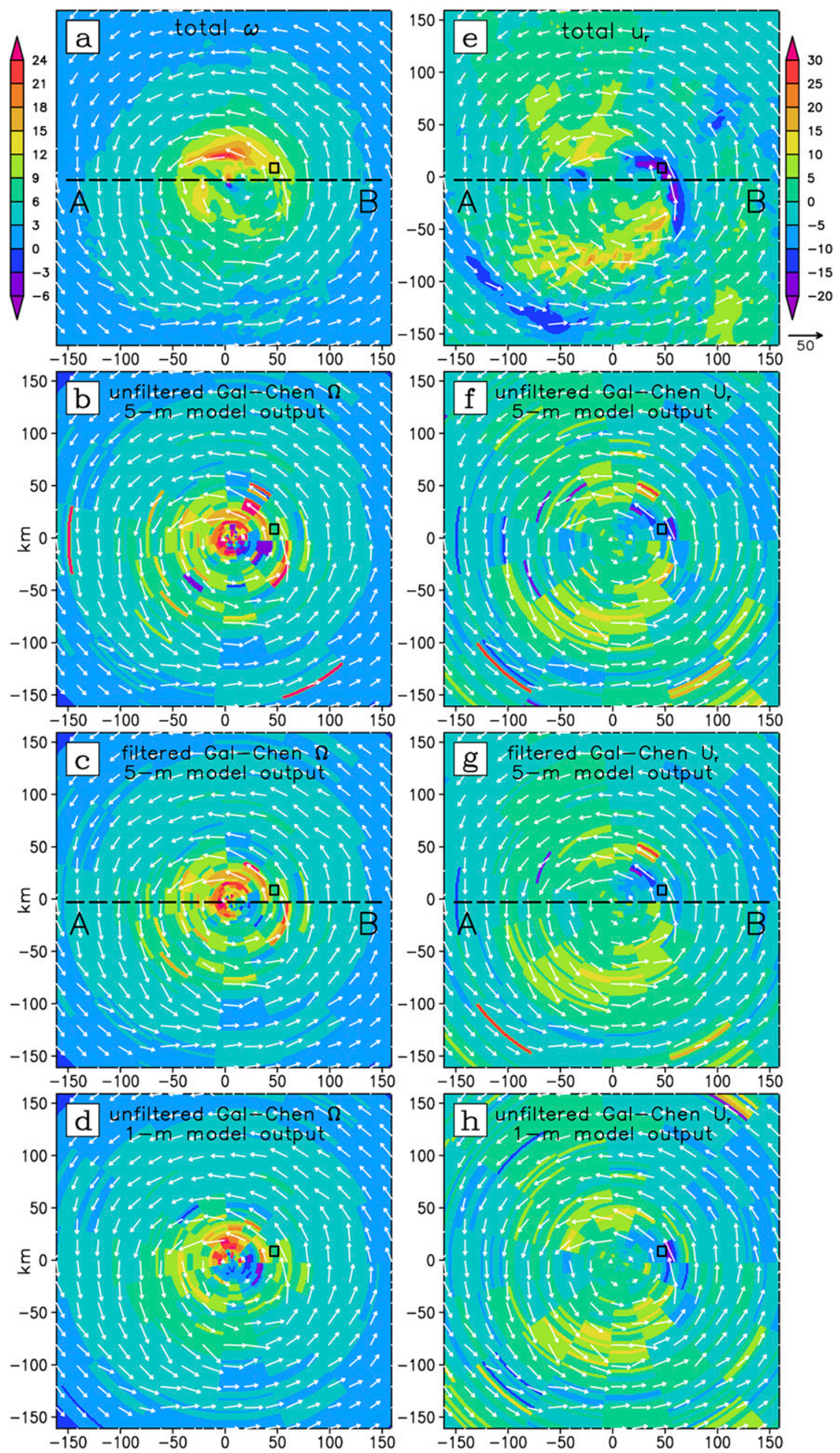

FIG. 9. (a) Distribution of the model-output total angular velocity $(\omega)$ (shaded, $\times 300^{\circ} \mathrm{s}^{-1}$ ) with storm-relative horizontal flow vectors $\left(\mathrm{m} \mathrm{s}^{-1}\right)$ at $z=6 \mathrm{~km}$ from the Hurricane Joaquin (2015) simulation at 2800; (b) as in (a), but for the advective component of angular velocity $(\Omega)$ found by the iterative Gal-Chen procedure using 5-min data output data; (c) as in (b), but after applying a filter to the Gal-Chen $\Omega$ field to remove local discontinuities, and (d) as in (b), but from the iterative Gal-Chen procedure using 1-min data. (e) As in (a), but for the total radial velocity $\left(u_{r}\right)\left(\mathrm{m} \mathrm{s}^{-1}\right)$. (f)-(h) As in (b)-(d), but for the advective component of the radial velocity $\left(U_{r}\right)\left(\mathrm{m} \mathrm{s}^{-1}\right)$. Dashed lines in (a), (c),(e), and (g) denote the vertical cross section shown in Fig. 10, and the black rectangular box corresponds to the region highlighted in Fig. 6. 

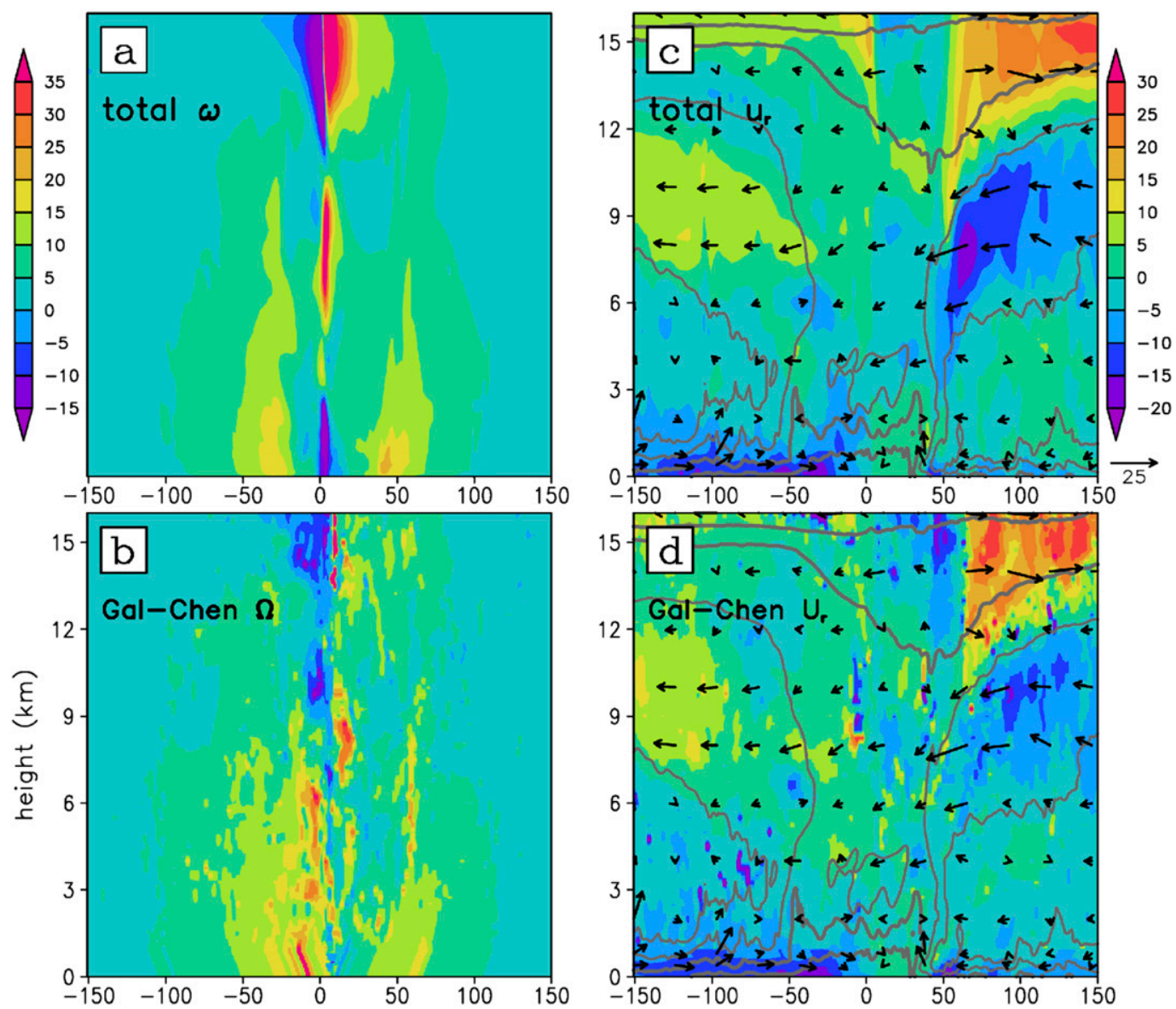

FIG. 10. (a) East-west vertical cross section of the model-output total angular velocity $(\omega)\left(\right.$ shaded, $\left.\times 300^{\circ} \mathrm{s}^{-1}\right)$ at the forecast time 2800 of the simulated Hurricane Joaquin (2015), running along the line AB shown in Fig. 9. (b) As in (a), but for the advective component of the angular velocity $(\Omega)$ found using the iterative Gal-Chen procedure over the preceding 5-min data output interval and then applying a filter to remove local discontinuities. (c) As in (a), but for the model-output total radial velocity $\left(u_{r}\right)$ (shaded, $\mathrm{m} \mathrm{s}^{-1}$ ), with in-plane flow vectors $\left(\mathrm{m} \mathrm{s}^{-1}\right.$; vertical velocity multiplied by 5) and $\theta_{e}(\mathrm{~K} ; 350 / 354$ thin-contoured, 358/362 thick-contoured). (d) As in (b), but for the advective component of the radial velocity $\left(U_{r}\right)\left(\mathrm{m} \mathrm{s}^{-1}\right)$. Distances from the 1-km model domain center $(\mathrm{km})$ are shown on the abscissa.

(cf. Figs. 6b,d and 8b). By replacing $\mathrm{AC}_{\mathrm{W}}$ with $\mathrm{AC}_{\mathrm{Uvw}}$, the mean $\triangle \mathrm{IDE}_{\mathrm{HORIZ}}$ increases from 1.42 to $4.44 \mathrm{~km}$, and the number of trajectories with $\Delta \mathrm{IDE}_{\mathrm{HORIZ}}>$ $10 \mathrm{~km}$ increases from 1030 to 1581 (Table 2). Interestingly, $\mathrm{AC}-\mathrm{MW}_{\mathrm{UVW}}$ slightly outperforms $\mathrm{AC}_{\mathrm{UVW}}$ in these statistics; similar trends can be found for $\triangle \mathrm{IDE}_{\text {VERT }}$ (Table 2).

\section{b. Evaluation of the iterative $U_{r}$ and $\Omega$ solver}

Let us now examine the advective flows $U_{r}$ and $\Omega$ retrieved by the iterative Gal-Chen-based solver for the 5- and 1-min Joaquin (2015) input data. In particular, we are interested in 1) how often the algorithm fails to converge, 2) the solution nonuniqueness threats, and 3) how these flows might differ from the model-output local winds. Considering all subdomains over the
2400-2800 period, the nonconvergence frequency is $3.28 \%$ for 5 -min data and near zero $(<0.01 \%)$ for 1 -min data; for cases of nonconvergence, $U_{r}$ and $\Omega$ are set to the subdomain-averaged flows ${ }^{7}$.

Taking a horizontal plan view of 2800 model-output winds at $z=6 \mathrm{~km}$, we find a markedly asymmetric pattern, with $\omega$ stronger in the northern eyewall (Fig. 9a), and a wavenumber-2 inflow-outflow pattern in $u_{r}$ (Fig. 9e). $U_{r}$ and $\Omega$ retrieved from the 5-min data

\footnotetext{
${ }^{7}$ Subdomain advections are also set to the first-guess values when the iterative Gal-Chen-based procedure converges upon physically unreasonable solutions, where $U_{r}$ exceeds $40 \mathrm{~m} \mathrm{~s}^{-1}$; these instances are quite rare, however, with a $\sim 0.1 \%$ occurrence rate for the 5-min data.
} 


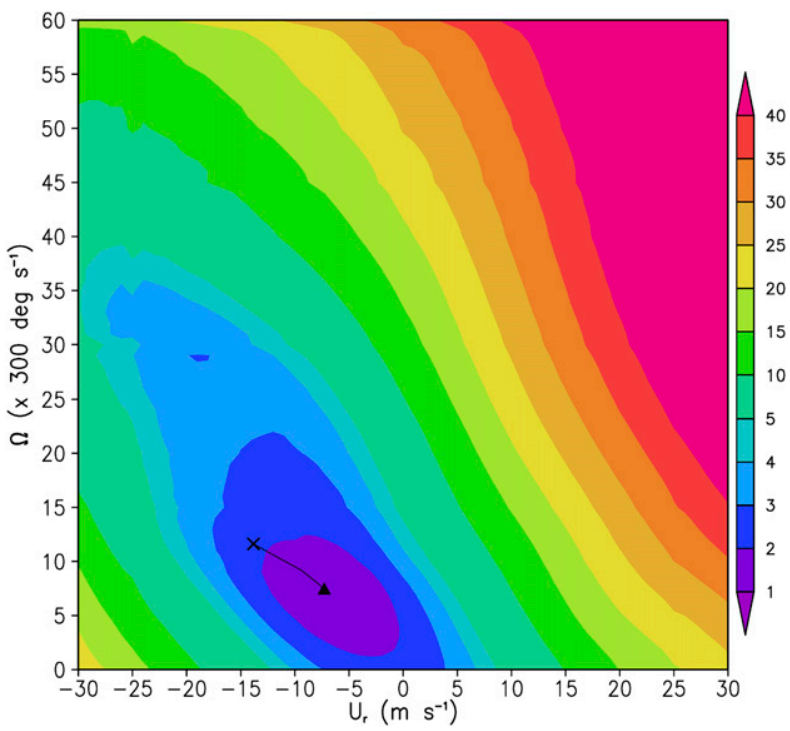

FIG. 11. As in Fig. 4, but the cost function $J\left(U_{r}, \Omega\right)\left(\mathrm{m}^{6} \mathrm{~s}^{-3} \times\right.$ $9 / \pi \times 10^{15}$ ) evaluated for a $z=6 \mathrm{~km}$ subdomain enclosed within the boxed region shown in Fig. 6, computed over the 5 -min time interval preceding forecast time 2800 .

(Figs. 9b,f) are generally similar to $u_{r}$ and $\omega$, although they contain a number of sharp local discontinuities that likely represent spurious solutions. Many of these discontinuities are eliminated using a filter applied to the 3D $U_{r}$ and $\Omega$ fields (Figs. 9c,g). In the first pass, the filter identifies all subdomains where either $U_{r}$ or $\Omega$ differ by more than $20 \%$ from a local average computed from the 26 surrounding subdomains in $(r, \lambda, z)$ space. The flagged "discontinuity subdomains" are then assigned $U_{r}$ and/or $\Omega$ values averaged from surrounding nonflagged subdomains in the second pass. All $\mathrm{AC}_{\mathrm{W}}$ and $\mathrm{AC}_{\mathrm{Uvw}}$ experiments described here use filtered advective flows. Finally, note the smoother unfiltered $U_{r}$ and $\Omega$ patterns retrieved from the 1-min data, compared to the 5-min data (cf. Figs. 9b,f, and 9d,h). These differences are consistent with our analytical tests (section 3 ) showing that spurious solutions move farther away from the true solution for smaller data input intervals; therefore, the iterative Gal-Chen-based procedure should be less likely to converge upon them, given reasonable first guesses. S15 found a similar result in their numerically simulated data tests (see their Figs. 16 and 18).

A vertical cross section taken through the 5-min filtered $U_{r}$ and $\Omega$ fields (Fig. 10) indicates that the advective flow structures are 1) vertically coherent despite being solved for one level at a time, and 2) generally similar in structure to the total flows in terms of the primary (Figs. 10a,b) and secondary circulations (Figs. 10c,d). One exception to item 2 is the aforementioned descending inflow channel for $r=50-100 \mathrm{~km}$, $z=6-10 \mathrm{~km}$. Here, $\left|U_{r}\right|$ is smaller than $\left|u_{r}\right|$, suggesting that local perturbation inflows $u_{r}^{\prime}$ may be present. Examining $J\left(U_{r}, \Omega\right)$ computed for a representative subdomain in this region (Fig. 11), we see that the global $J$-minimum does in fact correspond to a $\left|U_{r}\right|$ roughly $50 \%$ smaller than the first-guess $\left|u_{r}\right|$, and that the iterative Gal-Chen-based procedure successfully locates this global minimum. No problematic spurious local minima are present nearby.

\section{c. Remarks}

To better understand why LI-5 trajectory errors in the northeastern inner eyewall are substantially reduced by $\mathrm{AC}_{\mathrm{UVw}}$ but not by $\mathrm{AC}_{\mathrm{W}}$ (Figs. 6a,c), let us examine the time evolution of Joaquin's $z=6-\mathrm{km}$ horizontal winds over three successive 5-min output times, as shown in Fig. 12. Wind and relative vorticity anomalies are computed with respect to local time averages over the 1-h period centered on 2800. We find three distinct positive vorticity anomalies rotating cyclonically around the inner eyewall; these structures resemble the mesovortices found in the simulated Hurricane Bonnie (1998) by Braun et al. (2006). They translate between model output times over distances similar to their $\sim 10$-km horizontal scale. The horizontal velocity field otherwise remains quite stable over the 2730-2830 period. IDE $_{\text {HORIZ,LI }}$ is largest in the region transited by these disturbances, where it ranges from $10 \mathrm{~km}$ to as high as $70 \mathrm{~km}$ locally (not shown), suggesting that LI-5 is poorly estimating the local winds here. The widespread $\mathrm{AC}_{\mathrm{UVW}}$ error reduction found here suggests that $\mathrm{AC}$ of the horizontal winds improves the local wind estimates.

The slight improvement of $\mathrm{AC}-\mathrm{MW}_{\mathrm{UVw}}$ over $\mathrm{AC}_{\mathrm{UVW}}$ in terms of bulk sample statistics (Table 2) suggests that the subdomain-averaged flows are a reasonably good estimate of the advective flows for most portions of the simulated Joaquin (2015) circulation. Horizontal and vertical cross sections comparing $\left(u_{r}, \omega\right)$ and $\left(U_{r}, \Omega\right)$ support this conclusion (Figs. 9 and 10). However, we might expect the relative performance of the $\mathrm{AC}-\mathrm{MW}_{\mathrm{UVW}}$ and $\mathrm{AC}_{\mathrm{UVW}}$ methods to depend on a number of factors, including the TC case, region of interest, subdomain size, and model output time resolution. For example, even though S15 found that $\mathrm{AC}$ and $\mathrm{AC}-\mathrm{MW}$ yielded similar mean trajectory errors when the subdomains were kept sufficiently small, they also found that AC-MW performed quite poorly in the mesocyclone inflow region, where the local and pattern-translation $u$-wind speeds differed by $25 \mathrm{~m} \mathrm{~s}^{-1}$.

\section{Hurricane Wilma (2005) tests}

In this section, we investigate the utility of $\mathrm{AC}$ for computing trajectories through TC updraft cores, using 

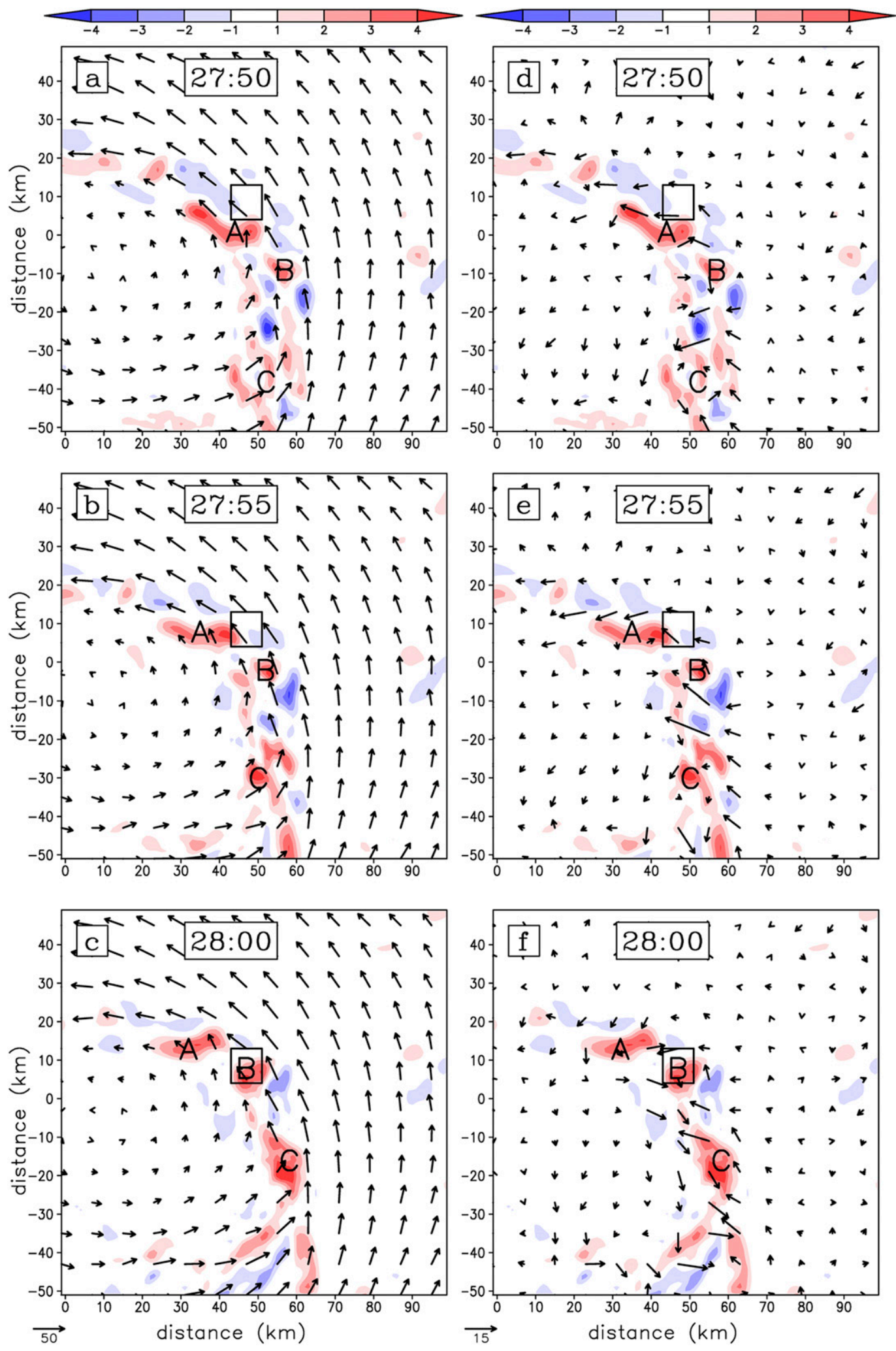

FIG. 12. (a) Distribution of the horizontal storm-relative flow vectors (at forecast time 2750), and the vertical component of relative vorticity anomaly $(\partial v / \partial x-\partial u / \partial y)^{\prime}$ (shaded, $\times 10^{-3} \mathrm{~s}^{-1}$ ), measured with respect to the local temporal average over the forecast period 2730-2830 from the Hurricane Joaquin (2015) WRF simulation 1-km resolution grid at $z=6 \mathrm{~km}$. (b),(c) As in (a), but for forecast times 2755 and 2800, respectively. (d)-(f) As in (a)-(c), but with perturbation flow vectors measured with respect to the local temporal average over the forecast period 2730-2830. Letter labels track cyclonic flow perturbations embedded in the mean flow. 

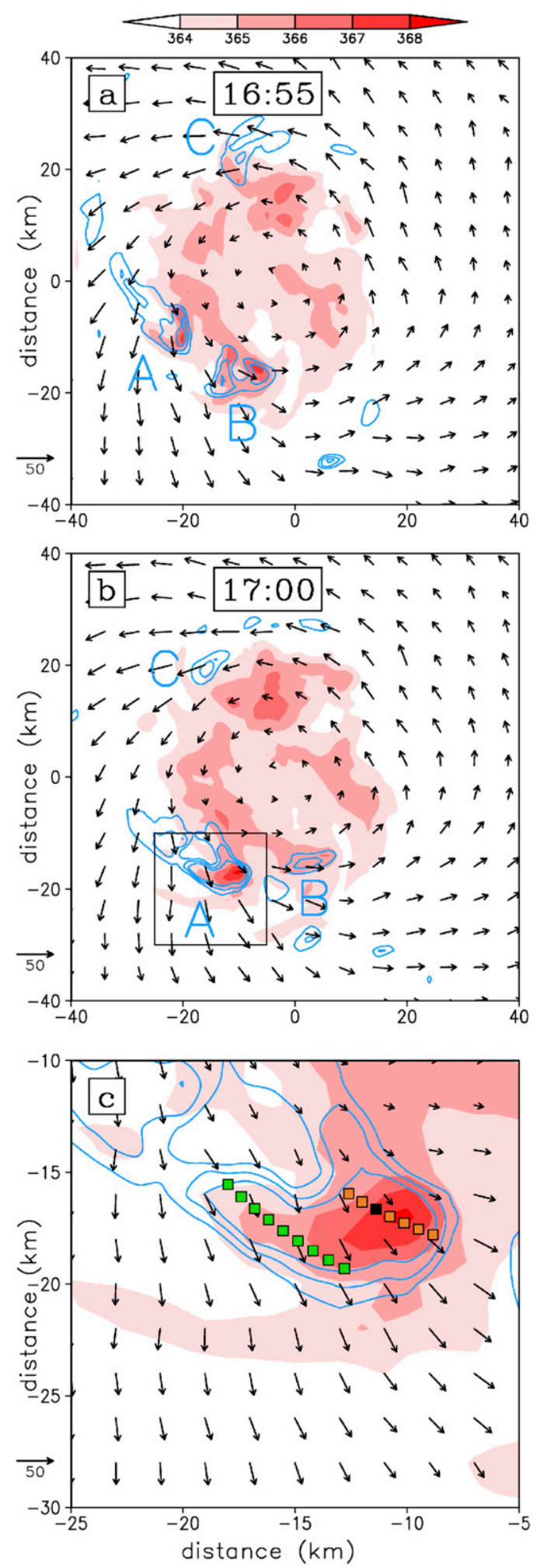

FIG. 13. (a) Distribution of $\theta_{e}$ (shaded, K), vertical velocity (contoured at $5 / 10 / 15 \mathrm{~m} \mathrm{~s}^{-1}$ ), and in-plane flow vectors $\left(\mathrm{m} \mathrm{s}^{-1}\right.$ ) taken from the Hurricane Wilma (2005) prediction at $z=14 \mathrm{~km}$ for forecast time 1655. Letter labels denote convective burst elements. the WRF-simulated Hurricane Wilma (2005) 5-min output data (Chen et al. 2011). Wilma's RI onset and upper-level warm core development coincide with an outbreak of particularly intense, well-defined CBs $\left(w>15 \mathrm{~m} \mathrm{~s}^{-1}\right)$ that rotate around the eyewall with lifetimes of $\sim 30 \mathrm{~min}$ (see Fig. 5 in CZ13). Only 5-min output was available for this case. However, an analysis of the advective flows retrieved by the Gal-Chen procedure over the 4-h backward trajectory computation period (not shown) indicates that they were generally similar to the local winds, with an overall nonconvergence rate of $5.7 \%$. To compare the same trajectories generated using $\mathrm{LI}$ and $\mathrm{AC}$, we examine along-trajectory traces of $w$ and $\theta_{e}$. Assuming saturated conditions, parcel $\theta_{e}$ is conserved after neglecting mixing, hydrometeor heat retention, and the latent heat of fusion. Our objective here is to show how artificial oscillations in parcel $w$ and $\theta_{e}$ result when LI is applied to rotating updraft cores sampled at less-than-ideal temporal resolution, and how AC can mitigate this problem.

Consider CB "A" shown in Fig. 13. While rotating about $30^{\circ}$ counterclockwise over the $1655-1700$ period, the updraft has broadened and intensified; note how the strongest portion moves in concert with a positive $\theta_{e}$ anomaly-possibly an artifact of intense fusion latent heat release (LHR) (Miller et al. 2015). Figure 14a shows a cluster of 4-h forward and 4-h backward AC trajectories seeded from inside CB "A" at $z=14 \mathrm{~km}$ (Fig. 13c). For comparison, a few trajectories representative of the background secondary circulation, with maximum $w$ not exceeding $7.5 \mathrm{~m} \mathrm{~s}^{-1}$, are shown in Fig. 14b. Both CB and "non-CB" updrafts originate from MBL parcels spiraling inward, with their $\theta_{e}$ increasing due to wind-induced ocean surface latent and sensible heat fluxes, consistent with the WISHE hypothesis (Emanuel 1986). CB and non-CB parcels both experience a $\theta_{e}$ reduction with ascent into the middle levels, likely from dry air entrainment (Cram et al. 2007), followed by a $\theta_{e}$ recovery in the upper troposphere, possibly on account of fusion heating (Fierro et al. 2009, 2012; Miller et al. 2015). Note the more pronounced $\theta_{e}$ "dip" extending through a deeper layer for the secondary circulation cluster; this is consistent with reduced upper-level fusion LHR in their comparatively weaker updrafts.

(b) As in (a), but for forecast time 1700. (c) As in (b), but zoomed in to show the seed positions (colored squares) of the convective burst trajectories. Horizontal distances are measured from the $1-\mathrm{km}$ resolution model domain center. 

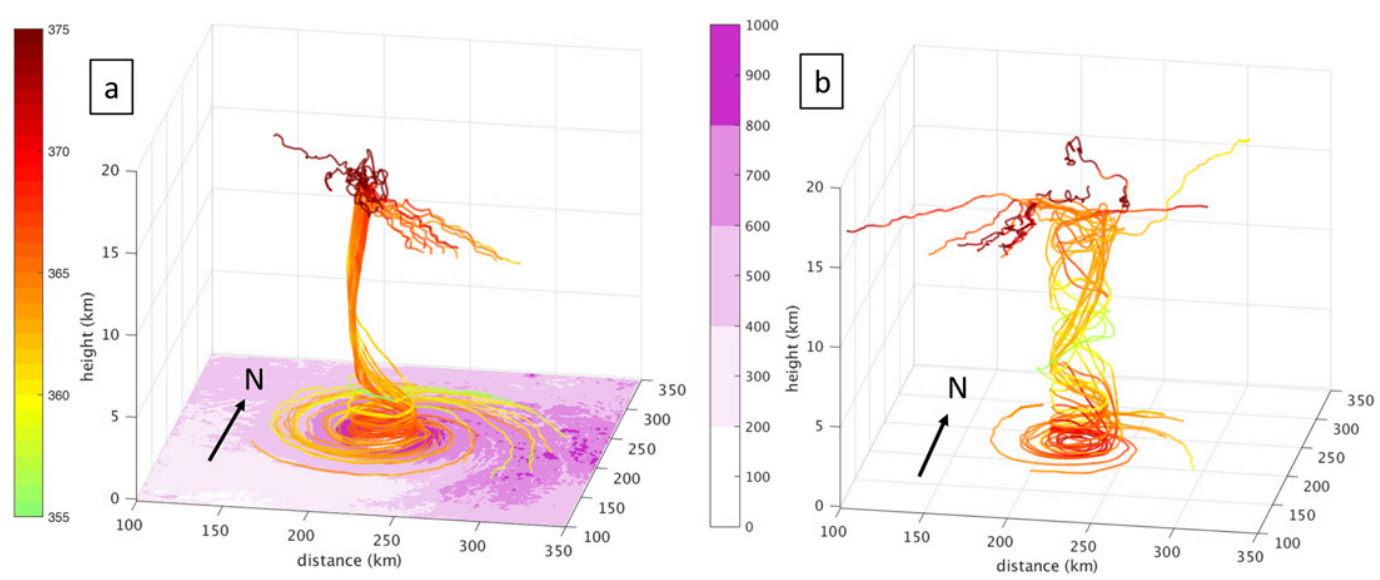

FIG. 14. Three-dimensional plots showing 8-h trajectories run from the Hurricane Wilma (2005) WRF prediction 5-min output, using advection correction time interpolation of the vertical velocity field $\left(\mathrm{AC}_{\mathrm{W}}\right)$. Four-hour forward and 4-h backward trajectories are seeded from $z=14 \mathrm{~km}$ at 1700 . (a) A cluster of 16 trajectories seeded from inside convective burst "A" in Fig. 13, and (b) 13 representative background secondary circulation trajectories. Lines are colored by $\theta_{e}(\mathrm{~K})$ interpolated along each trajectory. Purple shading in (a) shows the total (latent + sensible) heat flux $\left(\mathrm{W} \mathrm{m}^{-2}\right)$ from the ocean surface at forecast time 1500 . Horizontal distances are measured from the southwestern corner of the $1-\mathrm{km}$ resolution model domain.

To evaluate the impacts of AC, in Fig. 15 we compare $w, \theta_{e}$, and AAM along the $\mathrm{CB}$ "A" backward trajectories computed using $\mathrm{LI}, \mathrm{AC}_{\mathrm{W}}$, and $\mathrm{AC}_{\mathrm{UVw}}$. Note the prominent $w$ and $\theta_{e}$ oscillations above $z=6 \mathrm{~km}$ for the LI trajectories (Figs. 15b,c). The AC trajectories, on the other hand, show a more gradual decrease in $w$ and $\theta_{e}$ with downward extent (cf. Figs. 15f,g and 15j,k). AAM is reasonably well conserved along most trajectories above $z=3 \mathrm{~km}(z=1 \mathrm{~km})$ for LI (AC) (Figs. 15d,h,l). Previous TC AAM budgets using azimuthally averaged variables have found friction and turbulence forcing to be small above the MBL (Zhang et al. 2001; Qin et al. 2018). Our results suggest that the $3 \mathrm{D}$ AAM material conservation assumption used for the Gal-Chen-based $U_{r}$ and $\Omega$ solver was reasonable for this $\mathrm{CB}$.

Figure 16 replots $w$ and $\theta_{e}$ as a function of time along the first 10 min of the LI backward CB trajectories. Note how the oscillations of $w$ and $\theta_{e}$ generally stay in phase along each trajectory. Furthermore, $w$ and $\theta_{e}$ minima tend to occur in between the model output times, suggesting that they may be unphysical artifacts of the LI damping mechanism illustrated in Fig. 1. Between 1655 and 1700 , the $\sim 10^{\circ}$ wide $\mathrm{CB}$ " $\mathrm{A}$ " core region $(w>$ $15 \mathrm{~m} \mathrm{~s}^{-1}$ ) near $r=20 \mathrm{~km}$ rotates from $\sim 215^{\circ}$ to $\sim 245^{\circ}$ azimuth (Figs. 13a,b). The large "dip" in $w$ estimated along the LI trajectories around backward integration time $t=150 \mathrm{~s}$ (Fig. 16a) is consistent with $w(r=20 \mathrm{~km}$,

\footnotetext{
${ }^{8}$ Azimuthal-height plane cross sections (not shown) reveal that the CB " $A$ " updraft core remains nearly upright above the melting level at both 1655 and 1700 .
}

$\lambda=230^{\circ}$ ) being small for the two nearest model output times (Figs. 13a,b) ${ }^{8}$; this is simply a consequence of CB " $A$ " having a relatively small horizontal-scale relative to its translation speed. The absence of any significant local minima in the upper-level $\mathrm{AC}_{\mathrm{W}} w$ profiles (Fig. 15j), on the other hand, results in $\mathrm{AC}_{\mathrm{W}}$ bringing the parcels $\sim 2.5 \mathrm{~km}$ lower compared to LI after $20 \mathrm{~min}$ of backward integration (not shown).

\section{Summary and conclusions}

In this study, we present a new algorithm for computing trajectories from simulated TC data that interpolates the gridded winds in time using $\mathrm{AC}$ in lieu of traditional LI. Our goal is to reduce trajectory errors that result from inaccurate estimates of the wind field at the computational time step, which is typically smaller than the model output time resolution due to computational stability constraints. AC algorithms interpolate variables in time from a reference frame that moves with the advective flow velocity.

Our AC method extends the techniques previously developed in GC82 and S15 by 1) extending them to 3D flow fields, 2) applying the frozen turbulence constraint to AAM, and 3) defining the advective flows in cylindrical rather than Cartesian coordinates. We first tested our trajectory computation algorithms on an analytical flow field and determined that they are likely free of code errors. Next, we tested several alternate AC algorithms against LI using a batch of 10201 backward trajectories run from Hurricane Joaquin (2015) WRF simulation 5-min output, with 1-min output reserved for 

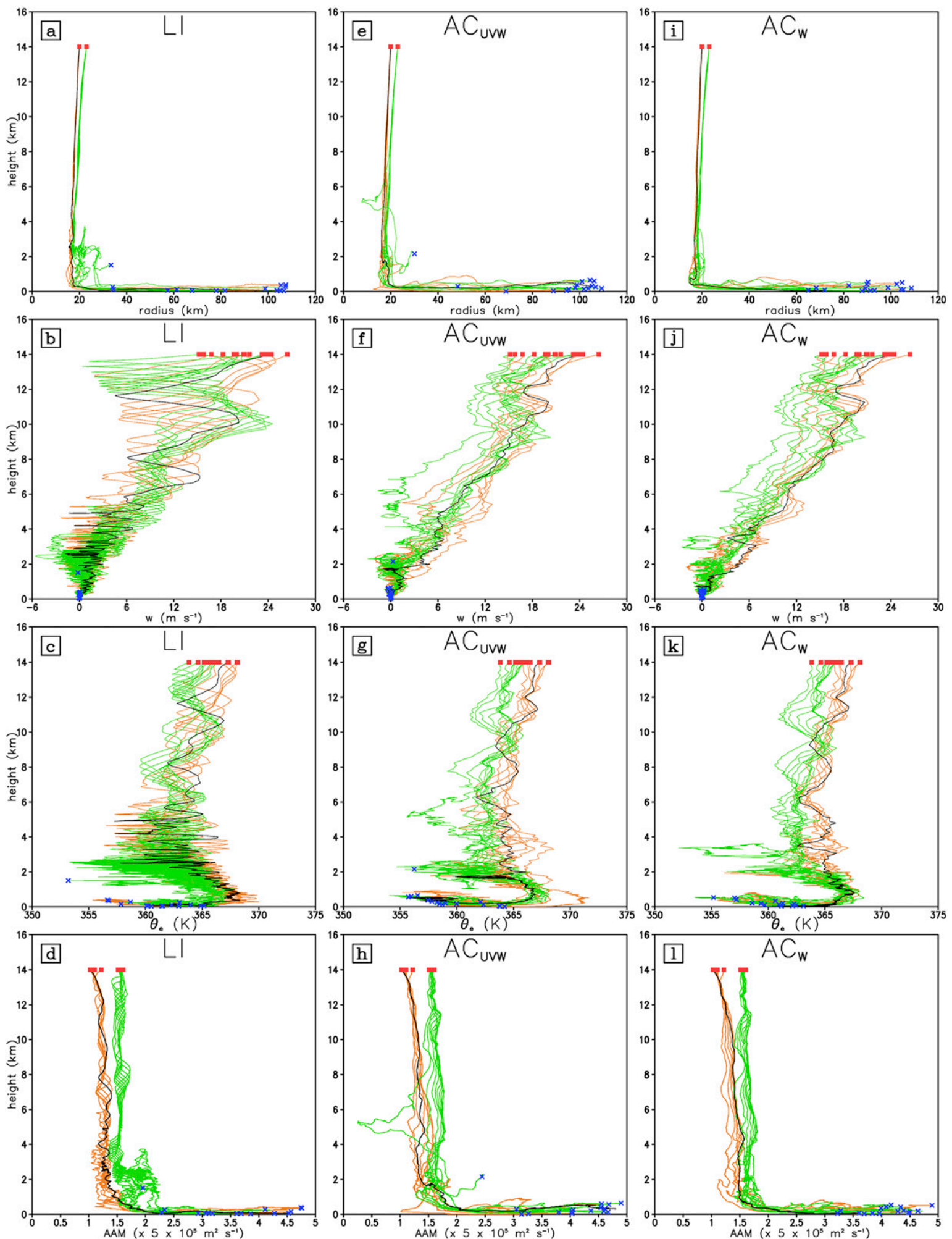

FIG. 15. (a) Radius-height projections of the 4-h backward CB "A" trajectories from the Hurricane Wilma (2005) WRF simulation (Fig. 13c), computed using linear interpolation (LI) in time. Orange (green) trajectories are seeded from the $r=20 \mathrm{~km}(r=23 \mathrm{~km})$ radius, and they correspond to the colored seed positions in Fig. 13c. Trajectory 3 is outlined in black. Red squares (blue crosses) denote seeded (final) positions. (b) Vertical velocity $\left(\mathrm{m} \mathrm{s}^{-1}\right.$ ) interpolated along the LI backward trajectories shown in (a). (c) As in (b), but for $\theta_{e}$ (K). (d) As in (b), but for AAM $\left(\times 5 \times 10^{5} \mathrm{~m}^{2} \mathrm{~s}^{-1}\right)$. (e)-(h) As in (a)-(d), but for the same trajectories rerun using advection correction of the horizontal and vertical velocity fields $\left(A C_{U V w}\right)$. (i)-(l) As in (a)-(d), but for the same trajectories rerun using advection correction of the vertical velocity field $\left(\mathrm{AC}_{\mathrm{W}}\right)$. 


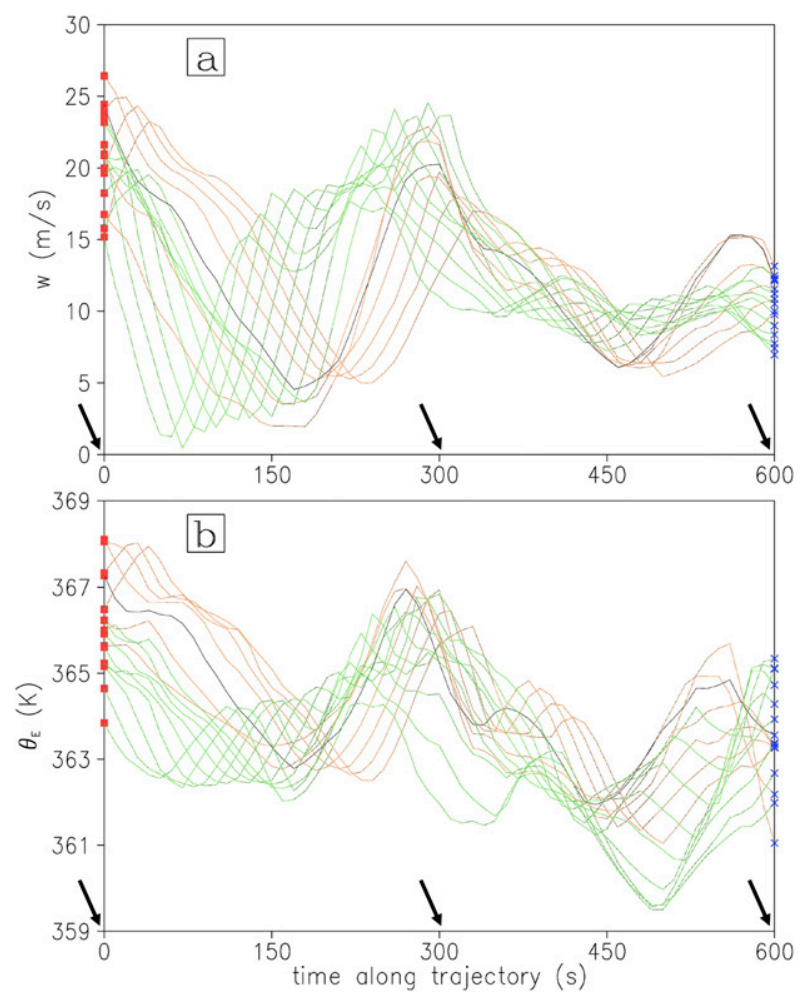

FIG. 16. (a) Vertical velocity $\left(\mathrm{m} \mathrm{s}^{-1}\right)$ plotted as a function of integration time for the $\mathrm{CB}$ " $\mathrm{A}$ " backward trajectories computed using LI, color coded as in Fig. 15. Arrows label model data output times. (b) As in (a), but for $\theta_{e}(\mathrm{~K})$.

computing reference, or "truth" trajectories. While AC of only $w$ led to little overall improvement over LI, AC of the full 3D wind vector $(u, v, w)$ improved trajectory accuracy more substantially, especially in the inner eyewall region where eyewall mesovortex structures were being advected by the tangential winds. Unlike S15, we obtained slightly better results when we defined the advective flows as gridpoint averages local to each subdomain, as opposed to using an iterative algorithm that minimized a cost function penalizing deviations from material conservation of AAM. This result was consistent with our findings that the iterative algorithm 1) retrieved advective flows that did not differ substantially from the model-output total winds over much of the model domain, and 2) occasionally converged on spurious solutions, with the likelihood increasing with coarser data input resolution. Finally, we showed how AC could eliminate along-trajectory $w$ oscillations that appear when LI is applied to rotating deep convective updrafts in a Hurricane Wilma (2005) WRF simulation; these oscillations likely result from the model output frequency being too coarse relative to the horizontal scale and translation speed of the updraft disturbances.

These results suggest that AC can improve the accuracy of trajectories computed from TC simulation output, particularly in regions where flows are unsteady over time, such as in eyewall mesovortices and convective bursts. Like S15, we recommend that AC be applied with caution, as there may be some TC cases, or at least portions of their circulations, where neither subdomainaveraged winds nor the iterative Gal-Chen-based procedure could yield reasonably accurate advective flows. Ideally, AC should be tested against LI using a set of reference trajectories computed at model runtime, or from higher-resolution data. Otherwise, the quality of the advective flow solution may be examined using analyses similar to those shown in section $4 b$ herein.

More generally, our findings motivate the testing of AC in trajectory calculations for meteorological applications beyond TCs; for these cases, better results would likely be obtained if the horizontal advective flows were defined in Cartesian rather than cylindrical coordinates, as in S15. It may also be worthwhile to test the impact of advection-correcting variables in the vertical direction. Here, vertical advections could be defined either by minimizing a cost function with respect to 3D flows, as in Zhang and Gal-Chen (1996), or simply by setting them to a locally averaged vertical velocity. In a forthcoming study, we will apply this $3 \mathrm{D}$ trajectory algorithm to a variety of flow structures using high-resolution WRF simulated TCs.

Acknowledgments. This work was funded by the U.S. Office of Naval Research Grants N000141410143, and N000141712210. The authors acknowledge the University of Maryland supercomputing resources (http:// hpcc.umd.edu) made available for conducting the research reported in this paper.

\section{APPENDIX A}

\section{Technique for Finding the Storm Center}

Planar views of the simulated Joaquin (2015) horizontal wind and relative vorticity fields reveal a center that tilts $\sim 25 \mathrm{~km}$ southward between the $z=3-\mathrm{km}$ and $z=8-\mathrm{km}$ levels over the trajectory computation period (not shown). The technique described below determines the storm center at every height over a user-selected layer for all model output times. The layer bottom (top) center coordinates are assigned to all heights below (above) the layer on the computational grid; subsequently, model-output winds are interpolated to cylindrical coordinates.

A first-guess storm center location for the lowest height is set to the centroid of the region where the sea level pressure is within $10(5) \mathrm{hPa}$ of $P_{\mathrm{MIN}}$ when the latter is less than (greater than) $990 \mathrm{hPa}$. Following 
TABLE A1. Wall-clock time and maximum combined processor memory usage for several configurations of an experiment running 10201 4-h backward trajectories on a 20-processor compute node. Input data is taken from the Hurricane Joaquin (2015) simulation and the $\mathrm{AC}_{\mathrm{UVw}}$ experiments apply advection correction below $z=16 \mathrm{~km}$ to the subdomain configuration described in section $2 \mathrm{c}$.

\begin{tabular}{lccccc}
\hline $\begin{array}{c}\text { Time interpolation } \\
\text { scheme }\end{array}$ & $\begin{array}{c}\text { Input data } \\
\text { resolution (min) }\end{array}$ & $\begin{array}{c}\text { Variables interpolated along } \\
\text { trajectories }\end{array}$ & $\begin{array}{c}\text { Shared memory } \\
\text { parallel processing }\end{array}$ & $\begin{array}{c}\text { Wall-clock } \\
\text { time (hhmm) }\end{array}$ & $\begin{array}{c}\text { Maximum RSS } \\
\text { memory usage }(\mathrm{GB})\end{array}$ \\
\hline $\mathrm{LI}$ & 5 & $x, y, z$ & Yes & 0025 & 21 \\
$\mathrm{AC}$ & 5 & $x, y, z$ & Yes & 0227 & 35 \\
$\mathrm{AC}_{\mathrm{UVW}}$ & 1 & $x, y, z$ & Yes & 0528 & 188 \\
$\mathrm{AC}$ & $x, y, z, p, T, \mathrm{AAM}, \theta_{e}$, & Yes & 0645 & 459 \\
$\mathrm{LI}$ & 1 & $q_{v}, q_{\text {frz }}, q_{\text {liq }}$ (2) & No & 0155 & 21 \\
$\mathrm{AC}$ & 5 & $x, y, z$ & No & 3127 & 37 \\
\hline
\end{tabular}

${ }^{11}$ Resident set size (RSS) memory is the portion of the main memory (RAM) on the compute node used by the executable.

${ }^{12}$ The variables $q_{v}, q_{\mathrm{frz}}$, and $q_{\text {liq }}$ refer to the mixing ratios of water vapor, frozen hydrometeors, and liquid hydrometeors, respectively.

Cavallo et al. (2013), the vortex center is defined as the grid point within a specified search box surrounding the first guess with maximum circulation (or equivalently, area-averaged $z$ component of relative vorticity) within a user-defined circulation radius. Inner-core vorticity patterns for TCs resolved at high resolution can be quite noisy due to small-scale variability in the tangential winds. To generate a horizontally smooth vorticity field representative of the vortex-scale circulation, our algorithm computes horizontal wind gradients as centered differences across $20-\mathrm{km}$ distances and applies five cycles of a nine-point local smoother to the input storm-relative $u$ and $v$ winds and the output vorticity. Center coordinates for each height become the first guesses for computing the center position of the level above. Alternatively, the sea level pressure centroid can be used for all heights; this was done when computing trajectories from the more axisymmetric and upright Wilma (2005) simulated vortex.

The Joaquin (2015) experiments described in section 4a use the circulation-based center determining algorithm with a 50-km-wide center search box and a circulation radius that expands linearly with height from $40 \mathrm{~km}$ at $z=3$ to $70 \mathrm{~km}$ at $z=8 \mathrm{~km}$ to account for vortex broadening. Users may optionally have the program output the automatically determined vortex center coordinates. If the center cannot be reliably determined by either method, they may supply the program with an auxiliary input file containing the center coordinates obtained by visual inspection.

\section{APPENDIX B}

\section{Computational Considerations}

The trajectory computation software developed for this study is written with OpenMP directives and designed to be run on a high-performance computing cluster using shared memory parallelism across multiple processors on a single node. Although the code can be compiled and run as a serial program, parallel processing yields a significant speedup, especially when implementing AC (Table A1). For experiments that use very high temporal resolution (i.e., $1 \mathrm{~min}$ ) input data and/or interpolate many nonkinematic variables along trajectories, storing the four-dimensional model data arrays during program execution may necessitate using large amounts of processor memory (RAM), depending on the model domain size (Table A1). Should machine RAM capacity become a limiting constraint, program memory usage could be reduced by running a batch of trajectories as a series of sequential jobs over shorter integration periods, using end points as the seed points for the next integration.

\section{REFERENCES}

Baker, J., 2010: Cluster analysis of long range air transport pathways and associated pollutant concentrations within the UK. Atmos. Environ., 44, 563-571, https://doi.org/10.1016/ j.atmosenv.2009.10.030.

Berg, R., 2016: National Hurricane Center tropical cyclone report: Hurricane Joaquin (28 September-7 October 2015). National Hurricane Center Tech. Rep. AL112015, 36 pp., https:// www.nhc.noaa.gov/data/tcr/AL112015_Joaquin.pdf.

Bowman, K. P., J. C. Lin, A. Stohl, R. Draxler, P. Konopka, A. Andrews, and D. Brunner, 2013: Input data requirements for Lagrangian trajectory models. Bull. Amer. Meteor. Soc., 94, 1051-1058, https://doi.org/10.1175/BAMSD-12-00076.1.

Brankov, E., S. T. Rao, and P. S. Porter, 1998: A trajectoryclustering-correlation methodology for examining the longrange transport of air pollutants. Atmos. Environ., 32, 1525-1534, https://doi.org/10.1016/S1352-2310(97)00388-9.

Braun, S. A., 2002: A cloud-resolving simulation of Hurricane Bob (1991): Storm structure and eyewall buoyancy. Mon. Wea. Rev., 130, 1573-1592, https://doi.org/10.1175/1520-0493(2002) $130<1573$ :ACRSOH $>2.0$.CO;2.

, M. T. Montgomery, and X. Pu, 2006: High-resolution simulation of Hurricane Bonnie (1998). Part I: The organization of 
eyewall vertical motion. J. Atmos. Sci., 63, 19-42, https:// doi.org/10.1175/JAS3598.1.

Cavallo, S. M., R. D. Torn, C. Snyder, W. Wang, and J. Done, 2013: Evaluation of the Advanced Hurricane WRF data assimilation system for the 2009 Atlantic hurricane season. Mon. Wea. Rev., 141, 523-541, https://doi.org/10.1175/MWR-D12-00139.1.

Chen, H., and D.-L. Zhang, 2013: On the rapid intensification of Hurricane Wilma (2005). Part II: Convective bursts and the upper-level warm core. J. Atmos. Sci., 70, 146-162, https:// doi.org/10.1175/JAS-D-12-062.1.

,,-- J. Carton, and R. Atlas, 2011: On the rapid intensification of Hurricane Wilma (2005). Part I: Model prediction and structural changes. Wea. Forecasting, 26, 885-901, https:// doi.org/10.1175/WAF-D-11-00001.1.

Cram, T. A., J. Persing, M. T. Montgomery, and S. A. Braun, 2007: A Lagrangian trajectory view on transport and mixing processes between the eye, eyewall, and environment using a high-resolution simulation of Hurricane Bonnie (1998). J. Atmos. Sci., 64, 1835-1856, https://doi.org/10.1175/ JAS3921.1.

Dahl, J. M. L., M. D. Parker, and L. J. Wicker, 2012: Uncertainties in trajectory calculations within near-surface mesocyclones of simulated supercells. Mon. Wea. Rev., 140, 2959-2966, https:// doi.org/10.1175/MWR-D-12-00131.1.

Dowell, D. C., and H. B. Bluestein, 2002: The 8 June 1995 McLean, Texas, storm. Part I: Observations of cyclic tornadogenesis. Mon. Wea. Rev., 130, 2626-2648, https://doi.org/10.1175/15200493(2002)130<2626:TJMTSP > 2.0.CO;2.

Doyle, J. D., and Coauthors, 2017: A view of tropical cyclones from above: The Tropical Cyclone Intensity (TCI) Experiment. Bull. Amer. Meteor. Soc., 98, 2113-2134, https://doi.org/ 10.1175/BAMS-D-16-0055.1.

Emanuel, K. A., 1986: An air-sea interaction theory for tropical cyclones. Part I: Steady-state maintenance. J. Atmos. Sci., 43, 585-604, https://doi.org/10.1175/1520-0469(1986)043<0585: AASITF $>2.0 . \mathrm{CO} ; 2$.

Fierro, A. O., J. Simpson, M. A. LeMone, J. M. Straka, and B. F. Smull, 2009: On how hot towers fuel the Hadley cell: An observational and modeling study of line-organized convection in the equatorial trough from TOGA COARE. J. Atmos. Sci., 66, 2730-2746, https://doi.org/10.1175/2009JAS3017.1.

, E. J. Zipser, M. A. LeMone, J. M. Straka, and J. Simpson, 2012: Tropical oceanic hot towers: Need they be undilute to transport energy from the boundary layer to the upper troposphere effectively? An answer based on trajectory analysis of a simulated TOGA COARE convective system. J. Atmos. Sci., 69, 195-213, https://doi.org/10.1175/JAS-D-11-0147.1.

Gal-Chen, T., 1982: Errors in fixed and moving frame of references: Applications for conventional and Doppler radar analysis. J. Atmos. Sci., 39, 2279-2300, https://doi.org/ 10.1175/1520-0469(1982)039<2279:EIFAMF>2.0.CO;2.

Gradshteyn, I. S., and I. M. Ryzhik, 2007: Table of Integrals, Series, and Products. 7th ed. Academic Press, 1171 pp.

Guimond, S. R., G. H. Heymsfield, and F. J. Turk, 2010: Multiscale observations of Hurricane Dennis (2005): The effects of hot towers on rapid intensification. J. Atmos. Sci., 67, 633-654, https://doi.org/10.1175/2009JAS3119.1.

Heymsfield, G. M., J. B. Halverson, J. Simpson, L. Tian, and T. P. Bui, 2001: ER-2 Doppler radar investigations of the eyewall of Hurricane Bonnie during the Convection and Moisture Experiment-3. J. Appl. Meteor., 40, 1310-1330, https://doi.org/ 10.1175/1520-0450(2001)040<1310:EDRIOT>2.0.CO;2.
Homeyer, C. R., K. P. Bowman, L. L. Pan, E. L. Atlas, R.-S. Gao, and T. L. Campos, 2011: Dynamical and chemical characteristics of tropospheric intrusions observed during START08. J. Geophys. Res., 116, D06111, https://doi.org/10.1029/ 2010JD015098.

Kaplan, J., and M. DeMaria, 2003: Large-scale characteristics of rapidly intensifying tropical cyclones in the north Atlantic basin. Wea. Forecasting, 18, 1093-1108, https://doi.org/10.1175/ 1520-0434(2003)018<1093:LCORIT>2.0.CO;2.

Kristiansen, N. I., and Coauthors, 2012: Performance assessment of a volcanic ash transport model mini-ensemble used for inverse modeling of the 2010 Eyjafjallajökull eruption. J. Geophys. Res., 117, D00U11, https://doi.org/10.1029/ 2011JD016844.

Kuo, Y.-H., M. Skumanich, P. L. Haagenson, and J. S. Chang, 1985: The accuracy of trajectory models as revealed by the observing system simulation experiments. Mon. Wea. Rev., 113 1852-1867, https://doi.org/10.1175/1520-0493(1985)113<1852: TAOTMA $>2.0 . \mathrm{CO} ; 2$.

Liu, Y., D.-L. Zhang, and M. K. Yau, 1999: A multiscale numerical study of Hurricane Andrew (1992). Part II: Kinematics and innercore structures. Mon. Wea. Rev., 127, 2597-2616, https://doi.org/ 10.1175/1520-0493(1999)127<2597:AMNSOH>2.0.CO;2.

Miller, W., and D.-L. Zhang, 2019: Understanding the unusual looping track of Hurricane Joaquin (2015) and its forecast errors. Mon. Wea. Rev., 147, 2231-2259, https://doi.org/ 10.1175/MWR-D-18-0331.1.

- H. Chen, and D.-L. Zhang, 2015: On the rapid intensification of Hurricane Wilma (2005). Part III: Effects of latent heat of fusion. J. Atmos. Sci., 72, 3829-3849, https://doi.org/10.1175/ JAS-D-14-0386.1.

Montgomery, M. T., and R. K. Smith, 2014: Paradigms for tropical cyclone intensification. Aust. Meteor. Oceanogr. J., 64, 37-66.

Onderlinde, M. J., and D. S. Nolan, 2016: Tropical cyclone-relative environmental helicity and the pathways to intensification in shear. J. Atmos. Sci., 73, 869-890, https://doi.org/10.1175/JASD-15-0261.1.

Pasch, R. J., E. S. Blake, H. D. Cobb III, and D. P. Roberts, 2006: Tropical cyclone report: Hurricane Wilma, 15-25 October 2005. NOAA/NHC Tech. Rep. AL252005, 27 pp., https:// www.nhc.noaa.gov/data/tcr/AL252005_Wilma.pdf.

Press, W. H., S. A. Teukolsky, W. T. Vetterling, and B. P. Flannery, 1992: Numerical Recipes in Fortran: The Art of Scientific Computing.Vol 1. 2nd ed. Cambridge University Press, 933 pp.

Qin, N., D.-L. Zhang, W. Miller, and C. Q. Kieu, 2018: On the rapid intensification of Hurricane Wilma (2005). Part IV: Inner-core dynamics during the steady RMW stage. Quart. J. Roy. Meteor. Soc., 144, 2508-2523, https://doi.org/10.1002/qj.3339.

Rogers, R. F., P. D. Reasor, and J. A. Zhang, 2015: Multiscale structure and evolution of Hurricane Earl (2010) during rapid intensification. Mon. Wea. Rev., 143, 536-562, https://doi.org/ 10.1175/MWR-D-14-00175.1.

Rössler, C. E., T. Paccagnella, and St. Tibaldi, 1992: A threedimensional atmospheric trajectory model: Application to a case study of Alpine lee cyclogenesis. Meteor. Atmos. Phys., 50, 211-229, https://doi.org/10.1007/BF01026018.

Shapiro, A., K. M. Willingham, and C. K. Potvin, 2010: Spatially variable advection correction of radar data. Part I: Theoretical considerations. J. Atmos. Sci., 67, 3445-3456, https://doi.org/ 10.1175/2010JAS3465.1.

- S. Rahimi, C. K. Potvin, and L. Orf, 2015: On the use of advection correction in trajectory calculations. J. Atmos. Sci., 72, 4261-4280, https://doi.org/10.1175/JAS-D-15-0095.1. 
Skamarock, W. C., and Coauthors, 2008: A description of the Advanced Research WRF version 3. NCAR Tech. Note NCAR/ TN-475+STR, 113 pp., https://doi.org/10.5065/D68S4MVH.

Stern, D. P., and F. Zhang, 2013: How does the eye warm? Part II: Sensitivity to vertical wind shear and a trajectory analysis. J. Atmos. Sci. 70, 1849-1873, https://doi.org/10.1175/JAS-D-12-0258.1.

Stewart, J., 1999: Multivariable Calculus. 4th ed. Brooks/Cole, 511 pp.

Stoelinga, M. T., 2009: A users' guide to RIP version 4.7: A program for visualizing mesoscale model output. UCAR, accessed 29 July 2019, http://www2.mmm.ucar.edu/wrf/users/ docs/ripug.htm.

Stohl, A., 1996: Trajectory statistics-A new method to establish source-receptor relationships of air pollutants and its application to the transport of particulate sulfate in Europe. Atmos. Environ., 30, 579-587, https://doi.org/10.1016/ 1352-2310(95)00314-2.

, and H. Kromp-Kolb, 1994: Origin of ozone in Vienna and surroundings, Austria. Atmos. Environ., 28, 1255-1266, https://doi.org/10.1016/1352-2310(94)90272-0.
G. Wotawa, P. Seibert, and H. Kromp-Kolb, 1995: Interpolation errors in wind fields as a function of spatial and temporal resolution and their impact on different types of kinematic trajectories. J. Appl. Meteor., 34, 2149-2165, https://doi.org/10.1175/ 1520-0450(1995)034<2149:IEIWFA>2.0.CO;2.

Taylor, G. I., 1938: The spectrum of turbulence. Proc. Roy. Soc. London, 164A, 476-490, https://doi.org/10.1098/ rspa.1938.0032.

— eddies from large ones. Proc. Roy. Soc. London, 158A, 499521, https://www.jstor.org/stable/96892.

Zhang, D.-L., Y. Liu, and M. K. Yau, 2001: A multiscale numerical study of Hurricane Andrew (1992). Part IV: Unbalanced flows. Mon. Wea. Rev., 129, 92-107, https://doi.org/10.1175/ 1520-0493(2001)129<0092:AMNSOH>2.0.CO;2.

Zhang, J., and T. Gal-Chen, 1996: Single-Doppler wind retrieval in the moving frame of reference. J. Atmos. Sci., 53, 2609-2623, https://doi.org/10.1175/1520-0469(1996)053<2609:SDWRIT> 2.0.CO;2. 